\title{
The effects of epistasis and linkage on the invasion of locally beneficial mutations and the evolution of genomic islands
}

April 30, 2021

Martin Pontz ${ }^{\mathrm{a}, \mathrm{b}, *}$ and Reinhard Bürger ${ }^{\mathrm{a}}$

a Department of Mathematics, University of Vienna, Austria

b Vienna Graduate School of Population Genetics

* Corresponding author

\section{Abstract}

We study local adaptation of a peripheral population by investigating the fate of new mutations using a haploid two-locus two-allele continent-island migration model. We explore how linkage, epistasis, and maladaptive gene flow affect the invasion probability of weakly beneficial de-novo mutations that arise on the island at an arbitrary physical distance to a locus that already maintains a stable migration-selection polymorphism. By assuming a slightly supercritical branching process, we deduce explicit conditions on the parameters that permit a positive invasion probability and we derive approximations for it. They show how the invasion probability depends on the additive and epistatic effects of the mutant, on its linkage to the polymorphism, and on the migration rate. We use these approximations together with empirically motivated distributions of epistatic effects to analyze the influence of epistasis on the expected invasion probability if mutants are drawn randomly from such a distribution and occur at a random physical distance to the existing polymorphism. We find that the invasion probability 
generally increases as the epistasis parameter increases or the migration rate decreases, but not necessarily as the recombination rate decreases. Finally, we shed light on the size of emerging genomic islands of divergence by exploring the size of the chromosomal neighborhood of the already established polymorphism in which $50 \%$ or $90 \%$ of the successfully invading mutations become established. These 'window sizes' always decrease in a reverse sigmoidal way with stronger migration and typically increase with increasing epistatic effect.

Key words: Local adaptation, Selection, Recombination, Invasion Probability, Gene Flow, Genetic Interactions, Branching process

\section{Introduction}

Local adaptation of a population to a new environment is frequently considered as a first step in a process leading to the emergence of a new species, because it increases the divergence between spatially separated populations. Often, gene flow will counteract this adaptive divergence. Local adaptation may occur from standing genetic variation or from new mutations. Gene flow is especially detrimental for a peripheral population if new, locally beneficial mutations need to be established to improve adaptation. Since a de-novo mutation starts out as a single copy, it is particularly prone to rapid loss caused by random events. As already shown by Haldane (1927) and Fisher (1930), the probability of survival of a mutant with selective advantage $s$ is approximately $2 s$. Maladaptive gene flow reduces or even annihilates this probability.

We study the influence of linkage and epistasis on the fate of a new, weakly beneficial mutation in a peripheral (island) population that is exposed to maladaptive gene flow from the main (continental) population. For this purpose, we extend a branching process model of Aeschbacher and Bürger (2014) by accounting for genetic interactions between loci. By a well known dichotomy, the mutant is either lost by random drift, which usually occurs rather quickly, or it invades and becomes established in the population. In this case, its further growth trajectory is (primarily) determined by selection in concert with other deterministic forces. Beneficial mutations of large effect are believed to be very rare. If their selective advantage exceeds the immigration rate, they have a positive establishment probability unless they arise in tight linkage to a deleterious background. As in Aeschbacher and Bürger (2014), we assume that at some 
locus there already exists a stable polymorphism in the island population, which was established because the selective advantage of the new (island) mutant at this locus exceeded the immigration rate of the ancestral (continental) allele. Thus, a first step in local adaptation has already been achieved.

We explore under which conditions such a polymorphic locus can act as a crystallization point for further adaptation. Because weakly beneficial mutations are more likely to occur than strongly beneficial mutations (e.g., Orr, 2010; Bataillon and Bailey, 2014; Rice et al., 2015), we focus on the case where the selective advantage of a new mutation on the island is smaller than that of the island mutant at the polymorphic locus. Then the genetic background in which the mutant occurs, and which contains the already established polymorphism, plays a crucial role in enabling invasion and survival of the mutant.

Previous studies (e.g., Aeschbacher and Bürger, 2014; Yeaman et al., 2016) investigated a similar question for diploids, however, with the restriction to a genic selection regime, i.e., by ignoring dominance and epistasis. They showed that the probability of establishment of the new mutation is always higher for loci that are tightly linked to the existing polymorphism than for loosely linked loci, although it is not always maximized for complete linkage. Their interpretation was that this process favors the emergence of genomic regions containing clusters of locally beneficial mutations (Yeaman and Whitlock, 2011), or at least of loci contributing to divergence. Such regions were dubbed genomic islands of divergence (Nosil et al., 2009; Feder and Nosil, 2010), of speciation (Turner et al., 2005), or of differentiation (Harr, 2006).

We extend and complement these results by taking into account genetic interactions between new locally beneficial mutations and the existing polymorphism. The invasion probability generally increases with more positive epistasis and mostly also with less recombination. However, it may be maximized at a positive recombination rate, especially if epistasis is positive and the additive effect not too small. Furthermore, the relative contributions of epistasis and linkage to the invasion probability depend crucially on the strength of gene flow. For weak migration epistasis is more efficient in increasing the invasion probability, whereas for strong migration linkage is more important.

Although we are in an era of relatively cheap sequencing technology and advanced bioinformatic tools, it is still unclear how epistatic values are distributed and which distribution is most prevalent in diploid organisms (e.g., Ehrenreich, 2017; Gao et al., 2010). More results are available for haploid organisms, because the fitness structure without dominance effects is simpler and also allows for mathematical approximations 
of the epistasis distribution (e.g., Martin et al., 2007; Blanquart et al., 2014; Schoustra et al., 2016). In order to take advantage of this fact and to limit mathematical complications, we investigate a haploid version of the general model.

Adaptation typically occurs on longer time scales, such that many new mutations may appear in a population, and their physical distance to the existing migrationselection polymorphism as well as their additive and epistatic effects on fitness may be considered as being drawn from appropriate distributions. Based on data-motivated distributions of the strength of epistasis, we especially analyze the expected effects of epistasis. By averaging the invasion probability of a mutation over such distributions, we can draw conclusions about the general importance of the involved evolutionary factors for the adaptation of a population and the expected genomic architecture. Concerning the latter, we follow Yeaman et al. (2016) and explore how the size of genomic islands of divergence will depend on migration rates, additive and epistastic effects, and their distributions.

\section{Methods}

\subsection{Model and biological scenario}

Our model is closely related to that employed by Aeschbacher and Bürger (2014), which is a diploid, discrete-time, two-locus two-allele model with continent-to-island (CI) migration. Whereas these authors assumed genic fitnesses, i.e., no dominance or epistasis, we include a parameter for genetic interaction between the two loci, but assume a haploid population.

The two loci are denoted by $\mathrm{A}$ and $\mathrm{B}$ and their alleles by $A_{1}, A_{2}$ and $B_{1}, B_{2}$. These form the four haplotypes $A_{1} B_{1}, A_{1} B_{2}, A_{2} B_{1}$, and $A_{2} B_{2}$, which occur at frequencies $x_{1}$, $x_{2}, x_{3}$, and $x_{4}$ on the island. We assume that the population on the continent is well adapted and fixed for alleles $A_{2}$ and $B_{2}$. The (im)migration rate to the island is denoted by $m$, i.e., each generation a fraction $m$ of the adult population (after selection and recombination) on the island is replaced by individuals of the continental population. The recombination rate between locus $\mathrm{A}$ and locus $\mathrm{B}$ is denoted by $r$, and the allele frequencies of $A_{1}$ and $B_{1}$ on the island are $p=x_{1}+x_{2}$ and $q=x_{1}+x_{3}$, respectively.

Initially, the island population is fixed for $A_{2}$, whereas at locus $\mathrm{B}$ the locally beneficial allele $B_{1}$ has arisen some time ago and is in migration-selection balance, which requires that its selective advantage $b$ exceeds the migration rate $m$ (see below). Then a weakly beneficial mutation occurs at locus $\mathrm{A}$, resulting in a single copy of $A_{1}$. Its fate is 
determined by direct selection on locus A, linkage to the selected locus B, migration, genetic interaction between the loci, and random genetic drift.

We focus on the scenario, where the island population is so large that after an initial stochastic phase during which the mutant is either lost or increases to appreciable frequency, the dynamics becomes deterministic. This implies, under some technical conditions, that the fate of $A_{1}$ is decided during the stochastic phase. If it survives this phase, it will reach either an attractor in the interior of the state space (most likely, a fully polymorphic equilibrium) or $B_{1}$ goes extinct but locus A stays polymorphic (see notebook $\mathrm{S} 1$ for details). This survival of the stochastic phase is what we synonymously call successful invasion or establishment.

\subsection{Fitness and evolutionary dynamics}

We use the following fitness scheme, which is general for a haploid two-locus two-allele model. The matrix $W=\left(w_{i j}\right)$ of relative genotypic fitnesses on the island is normalized such that

$$
W=\left(\begin{array}{cc}
B_{1} & B_{2} \\
1+a+b+e & 1+a \\
1+b & 1
\end{array}\right) \begin{gathered}
A_{1} \\
A_{2}
\end{gathered}
$$

where $a>0$ is the selective advantage of the new mutant $A_{1}$ relative to the resident (continental) type $A_{2}$ on the background $B_{2}$; thus, $A_{1} B_{2}$ produces on average $1+a$ times as many offspring as $A_{2} B_{2}$. Analogously, $b>0$ is the selective advantage of $B_{1}$ relative to $B_{2}$ on the background $A_{2}$. The parameter $e$ measures epistasis, i.e., the deviation of the fitness of $A_{1} B_{1}$ from additivity. The only restriction we pose on $e$ is $-1-a-b<e$, so that the fitness of $A_{1} B_{1}$ is positive. We have negative (positive) epistasis if $e<0(e>0)$.

The evolutionary dynamics of such a haploid model depends on the life cycle of the population. We investigate only the dynamics in which selection occurs first, followed by recombination and migration. For weak evolutionary forces, the dynamics becomes independent of the order of selection, recombination, and migration (e.g. Bürger, 2014), and most explicit formulas simplify (e.g., Sect. 3.2).

Because selection occurs before recombination, we define the measure $D$ of linkage disequilibrium by

$$
D=\frac{1}{\bar{w}^{2}}\left(w_{11} w_{22} x_{1} x_{4}-w_{12} w_{21} x_{2} x_{3}\right)
$$


Then the dynamical equations can be written as

$$
\begin{aligned}
x_{1}^{\prime} & =(1-m)\left(\frac{w_{11} x_{1}}{\bar{w}}-r D\right), \\
x_{2}^{\prime} & =(1-m)\left(\frac{w_{12} x_{2}}{\bar{w}}+r D\right), \\
x_{3}^{\prime} & =(1-m)\left(\frac{w_{21} x_{3}}{\bar{w}}+r D\right), \\
x_{4}^{\prime} & =(1-m)\left(\frac{w_{22} x_{4}}{\bar{w}}-r D\right)+m,
\end{aligned}
$$

where the mean fitness is given by

$$
\bar{w}=w_{11} x_{1}+w_{12} x_{2}+w_{21} x_{3}+w_{22} x_{4}
$$

(cf. Felsenstein, 2019, chapter "Selection and Recombination", p. 364).

Throughout this paper, we assume that the selective advantage $b$ of $B_{1}$ is large enough so that a stable polymorphism at $\mathrm{B}$ can be maintained in the island population independently of any other locus. A simple calculations shows that this is case if and only if $b>m /(1-m)$ or, equivalently, if

$$
0<m<m_{B}:=\frac{b}{1+b}
$$

(cf. Haldane, 1930; Wright, 1931). Then the equilibrium frequency of $B_{1}$ is

$$
q_{B}=\frac{b-m(1+b)}{b} .
$$

We call this equilibrium $E_{B}$.

As argued above, we assume throughout that if a new beneficial mutant $A_{1}$ occurs, it has a small fitness effect $a$, by which we mean $a<b$. We note that many of the tedious calculations performed to derive the results presented below are given in Mathematica notebooks (Wolfram Research 2020), which constitute the supplementary files S1 - S5.

\subsection{Two-type branching process}

We model the initial stochastic phase, after occurrence of $A_{1}$, by a two-type branching process in discrete time (Harris, 1963). The two types are the haplotypes $A_{1} B_{1}$ and $A_{1} B_{2}$. Depending on the first occurrence of the mutant on background $B_{1}$ or $B_{2}$, the invasion probability of $A_{1} B_{1}$ or $A_{1} B_{2}$ is denoted by $\pi_{1}$ or $\pi_{2}$, respectively. The probability that $A_{1}$ initially occurs in an individual with the $B_{1}$ background depends on the equilibrium frequency of $B_{1}$ at $E_{B}$, which is $q_{B}$ in (6). 
The (mean) invasion probability $\bar{\pi}$ of $A_{1}$ is thus the sum of the two conditional probabilities weighted by the frequencies of $B_{1}$ and $B_{2}$ at equilibrium:

$$
\bar{\pi}=q_{B} \pi_{1}+\left(1-q_{B}\right) \pi_{2}
$$

The invasion probability of $A_{1}$ depends crucially on the so-called mean matrix $\mathbf{M}$ which, for a two-type process, is the $2 \times 2$ matrix with entries $\lambda_{i j}$, where $\lambda_{i j}$ is the mean number of $j$-type offspring produced by an $i$-type parent in each generation (while the mutant $A_{1}$ is rare). The entries $\lambda_{i j}$ can be obtained from of our basic recursion system (3) by computing the Jacobian $J$ at the single-locus polymorphism $E_{B}$ and identifying $\mathbf{M}$ as the transposed of the left upper $2 \times 2$ submatrix of $J$, which describes the dynamics of $A_{1} B_{1}$ and $A_{1} B_{2}$ (for details, see File S1, Sect. 6). Thus, the linearized dynamics around the equilibrium $E_{B}$ pointing into the simplex is given by

$$
\left(x_{1}^{\prime}, x_{2}^{\prime}\right)=\left(x_{1}, x_{2}\right) \mathbf{M}
$$

where, by a simple computation and then by substituting (1) into (9a),

$$
\begin{aligned}
\mathbf{M} & =\frac{1-m}{\bar{w}_{B}^{2}}\left(\begin{array}{cc}
w_{11}\left(\bar{w}_{B}-r w_{22}\left(1-q_{B}\right)\right) & r w_{11} w_{22}\left(1-q_{B}\right) \\
r w_{12} w_{21} q_{B} & w_{12}\left(\bar{w}_{B}-r w_{21} q_{B}\right)
\end{array}\right) \\
& =\frac{1}{b(1+b)(1-m)}\left(\begin{array}{cc}
(1+a+b+e)[b(1-m)-m r] & (1+a+b+e) m r \\
(1+a)[b(1-m)-m] r & (1+a)[b(1-m)(1-r)+m r]
\end{array}\right) .
\end{aligned}
$$

Here, $\bar{w}_{B}=w_{21} x_{3}+w_{22} x_{4}=1+b q_{B}=(1+b)(1-m)$ is the mean fitness at $E_{B}$.

It is well known (e.g. Harris, 1963) that invasion occurs with positive probability if and only if the leading eigenvalue $\lambda$ of $\mathbf{M}$ satisfies

$$
\lambda>1
$$

\section{Analysis of the invasion condition}

To apply the above theory to our model, we compute the leading eigenvalue $\lambda$ of $\mathbf{M}$ and find (File S1, Sect. 6)

$$
\lambda=\frac{b(2+2 a+b+e)(1-m)-[b(1+a(1-m))+e m] r+\sqrt{R}}{2 b(1+b)(1-m)},
$$

where

$$
\begin{aligned}
R= & b^{2}(b+e)^{2}(1-m)^{2}-2 b(b+e)(1-m)[(2+2 a+2 b+a b+e) m-(1+a) b] r \\
& +\left[e m+b(1+a(1-m)]^{2} r^{2} .\right.
\end{aligned}
$$


Using (11), we can rewrite the invasion condition (10) in the form

$$
L<\sqrt{R}
$$

where

$$
L=2 b(1+b)(1-m)-b(2+2 a+b+e)(1-m)+[b(1+a(1-m))+e m] r .
$$

It will be useful to define $\phi=\left(R-L^{2}\right) /[4 b(1-m)]$, which simplifies to

$$
\begin{aligned}
\phi= & b(b-a)(a+e)(1-m) \\
& +r\left\{b(1+a)(a+e)-\left[b^{2}+b\left(1+a+a^{2}\right)+(1+2 b+a b) e\right] m\right\} .
\end{aligned}
$$

Note that $4 b(1-m)>0$ and thus does not influence the sign of $\phi$, which is linear in both $m$ and $r$. Therefore, the invasion condition (10) is satisfied if and only if $L<0$ or $\phi>0$. In File S2, Sect. 2, we show that $L<0$ implies $\phi>0$. As a consequence,

$$
\lambda>1 \text { if and only if } \phi>0 .
$$

\subsection{Characterization of the invasion condition in terms of the migration and the recombination rate}

According to (16), invasion of $A_{1}$ is possible if and only if $\phi>0$, where we recall our assumption $0<a<b$. Below, we define a critical migration rate $m^{*}$ and then, alternatively, a critical recombination rate $r^{*}$ to characterize the parameter region in which invasion of $A_{1}$ can occur. The detailed derivations of the following results can be found in File S2, Sect. 3. We start by defining

$$
e_{r}=-a+(1+b) \frac{r}{1-r}
$$

and

$$
m^{*}=\frac{b(a+e)[b-a+(1+a) r]}{b(b-a)(a+e)+\left[b^{2}+b\left(1+a+a^{2}\right)+e(2 b+a b+1)\right] r},
$$

and note that $e_{r}>0$ if and only if $r>\frac{a}{1+a+b}$. The following is our first main result.

\section{Proposition 1.}

(i) If $-1-a-b<e \leq-a$, then invasion is impossible.

(ii) If $-a<e<e_{r}$, then $0<m^{*}<m_{B}$ and invasion is possible if $m \in\left[0, m^{*}\right)$. 
(iii) If $e_{r} \leq e$, then invasion is possible if $m \in\left[0, m_{B}\right)$.

Proof. The proof can be simplified by transforming the linear function $\phi(m)$ in $(15)$ into a function $\hat{\psi}(z)$ by substituting $m=\frac{b z}{(1+b)(1+z)}$. This strictly monotone transformation maps the interval $\left[0, m_{B}\right)=\left[0, \frac{b}{1+b}\right)$ for admissible $m$ (such that $E_{B}$ exists) onto the interval $[0, \infty)$ for $z$. Then $\psi(z)=\frac{(1+z)(1+b)}{b} \hat{\psi}(z)$ is linear in $z$ and has the same sign as $\hat{\psi}(z)$. In fact, $\psi(z)=c_{0}+c_{1} z$, where

$$
c_{0}=(1+b)(a+e)(b-a+r(1+a))
$$

and

$$
c_{1}=(b-a)(a+e-(1+a+b+e) r)
$$

If $-1-a-b<e<-a$, then $c_{0}<0$ and $c_{1}<0$, and thus $\psi(z)<0$ if $z>0$. This settles (i).

If $-a<e<e_{r}$, then $c_{0}>0$ and $c_{1}<0$. Therefore, $\psi(z)>0$ if $z \in\left(0, z^{*}\right)$, where $z^{*}$ is the zero of $\psi(z)$. The corresponding zero of $\phi(m)$ is $m^{*}$ given by $(17 \mathrm{~b})$. In this case, $0<m^{*}<m_{B}$ is clearly satisfied and thus, (ii) holds.

If $e>e_{r}$, then $c_{0}$ and $c_{1}$ are positive. This implies that $\psi(z)>0$ if $z>0$, which in turn implies (iii).

As already noted above, $\phi$ is also a linear function in $r$ and thus we can reformulate Proposition 1 in terms of $r$. We define

$$
e_{m}=-a+\frac{(b-a)(1+b) m}{\left[(1+b)^{2}+b(b-a)\right](1-m)-(1+b)}
$$

and

$$
r^{*}=\frac{(1-m)(a+e)(b-a) b}{[b(1+b)+a(1+a) b+(1+2 b+a b) e] m-(1+a)(a+e) b},
$$

and note that $e_{m}>0$ if $m>a(1-a+2 b) /\left(1+a+b+2 a b-a^{2}\right)$, where the lower bound is close to $a$ if $a$ and $b$ are small. Now we can reformulate Proposition 1 as follows.

\section{Proposition 2.}

(i) If $-1-a-b<e \leq-a$, then invasion is impossible.

(ii) If $-a<e<e_{m}$, then $0<r^{*}<\frac{1}{2}$ and invasion is possible if $r \in\left[0, r^{*}\right)$.

(iii) If $e_{m} \leq e$, then invasion is possible if $r \in\left[0, \frac{1}{2}\right]$. 
Proof. With the transformation $r=\frac{z}{2(1+z)}$, the proof is similar to that for Proposition 1.

Of course, the parameter regions described by Propositions 1 and 2 are identical. However, it is useful to have these two representations, because we want to investigate how epistasis interacts with linkage and with migration in admitting invasion of $A_{1}$.

Remark 3. (i) The above propositions show that invasion of $A_{1}$ is possible only if there is no sign epistasis, i.e., if $a+e>0$, which means that the genotype $A_{1} B_{1}$ must have higher fitness in the fitness scheme (1) than the other genotypes.

(ii) Simple calculations (File S2, Sect. 3) show that if $0<m^{*}<m_{B}$, then $m^{*}$ is strictly increasing in $e$ and in $a$, and $m^{*}$ is strictly decreasing in $r$. In addition, $m^{*}=m_{B}$ if $e=e_{r}$.

(iii) Analogously, $r^{*}$ is strictly increasing in $e$ and in $a$, and it is decreasing in $m$ provided $e>-a$. In addition, $r^{*}=\frac{1}{2}$ if $e=e_{m}$.

(iv) Therefore, these propositions show that invasion of $A_{1}$ is always facilitated by larger $e$ and, of course, by larger $a$. Moreover, $e_{m}$ increases in $m$ and $e_{r}$ increases in $r$.

(v) We conclude that for fixed selection coefficients $a, b$, and $e$ satisfying $a+e>0$, a reduction in $r(m)$ will increase the maximum value of $m(r)$ for which invasion is possible.

(vi) The proof of Proposition 1 implies that $\lambda=1$ if and only if $m=m^{*}$ or, equivalently, $r=r^{*}$.

(vii) If $A$ and $B$ are completely linked $(r=0)$, then $\lambda$ is independent of $m$ and invasion of $A_{1}$ is possible for every $m$ if $a+e>0$.

\subsection{Weak-forces approximation}

Several of the above expressions are complicated and do not easily provide analytical insight. However, if we assume that all evolutionary forces are weak, then much simpler expression are obtained. For this purpose, we scale the parameters for selection, migration, and recombination as follows: $a \rightarrow \delta \alpha, b \rightarrow \delta \beta, e \rightarrow \delta \epsilon, m \rightarrow \delta \mu, r \rightarrow \delta \rho$, and assume that $\delta$ is small. By performing series expansions to first order in $\delta$ of the entries of the mean matrix $\mathbf{M}$ and returning to the original parameters (i.e., using roman font again), we obtain the weak-forces approximation

$$
\tilde{\mathbf{M}}=\left(\begin{array}{cc}
1+a+e-m r / b & m r / b \\
r(1-m / b) & 1+a-b-r(1-m / b)
\end{array}\right) .
$$



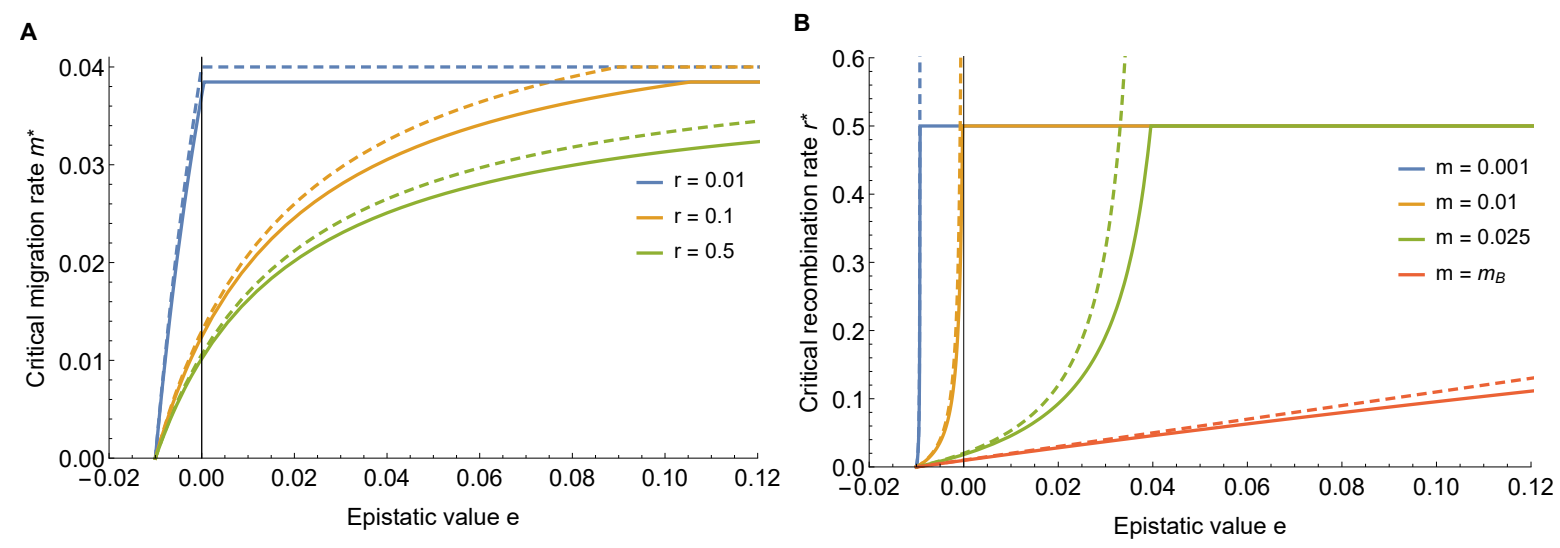

Figure 1: Critical migration and recombination rates, $m^{*}$ and $r^{*}$, below which invasion of the mutant $A_{1}$ can occur. The solid curves in panels $\mathrm{A}$ and $\mathrm{B}$ show the exact expressions for $m^{*}$ and $r^{*}$, given by (17b) and (19b), respectively, as functions of the epistatic value $e$. The dashed curves show the corresponding weak-forces approximations, which are given by $(22 \mathrm{~b})$ and $(22 \mathrm{~d})$. In both panels the other parameters are $a=0.01$ and $b=0.04 ;$ therefore, $m_{B} \approx 0.0385$.

By applying the same procedure to every $x_{i}^{\prime}-x_{i}$ in the recursion system (3), we obtain a system of approximating differential equations. Computing the Jacobian of this system at the equilibrium $E_{B}$, where in continuous time $x_{1}=x_{2}=0, x_{3}=\tilde{q}_{B}=1-m / b$, and $x_{4}=m / b$, we find that the upper left $2 \times 2$ submatrix is $\tilde{\mathbf{M}}-\mathbf{I}$, where $\mathbf{I}$ is the $2 \times 2$ identity matrix.

Throughout, we use the tilde to denote the weak-forces approximation. The leading eigenvalue of $\tilde{\mathbf{M}}$ is

$$
\tilde{\lambda}=1-\frac{1}{2}(b+r-2 a-e)+\frac{1}{2} \sqrt{(b+e)^{2}+2(b+e)\left(1-\frac{2 m}{b}\right) r+r^{2}} .
$$

Straightforward calculations show that the bounds used in Propositions 1 and 2 simplify to

$$
\begin{aligned}
m_{B} \approx \tilde{m}_{B} & =b, \\
m^{*} \approx \tilde{m}^{*} & =\frac{b(a+e)(b-a+r)}{(b+e) r} \\
e_{r} \approx \tilde{e}_{r} & =r-a, \\
r^{*} \approx \tilde{r}^{*} & =\frac{(b-a)(a+e) b}{(b+e) m-(a+e) b} \\
e_{m} \approx \tilde{e}_{m} & =-a+\frac{(b-a) m}{b-m} .
\end{aligned}
$$

With these definitions, the following weak-forces versions of Propositions 1 and 2 are easily derived, which yield simpler interpretations of the invasion conditions. 


\section{Proposition 4.}

(i) If $e \leq-a$, then invasion is impossible.

(ii) If $-a<e<r-a$, then $0<\tilde{m}^{*}<b$ and invasion is possible if $m \in\left[0, \tilde{m}^{*}\right)$.

(iii) If $r-a \leq e$, then invasion is possible if $m \in[0, b)$.

\section{Proposition 5.}

(i) If $e \leq-a$, then invasion is impossible.

(ii) If $-a<e<\tilde{e}_{m}$, then $\tilde{r}^{*}>0$ and invasion is possible if $r \in\left[0, \tilde{r}^{*}\right)$.

(iii) If $\tilde{e}_{m} \leq e$, then invasion is possible if $r \in[0, \infty)$.

Remark 3 applies to these propositions as well. The computations for Sect. 3.2 are presented in File S5, Sects. 1,2.

\section{Invasion probabilities}

So far, we have characterized the parameter regions in which invasion of the new mutant $A_{1}$ is possible, but we have not yet quantified the probability of invasion. In the following, we assume discrete time and that the number of offspring of type $j$ produced by an individual of type $i$ is Poisson distributed with mean $\lambda_{i j}$, where the $\lambda_{i j}$ are the entries of the matrix $\mathbf{M}$ given by (9b). Branching-process theory (e.g. Harris, 1963) states that the probabilities $\sigma_{1}=1-\pi_{1}$ and $\sigma_{2}=1-\pi_{2}$ of loss of type- 1 and type- 2 individuals, respectively, are given by the smallest positive solutions of

$$
\begin{aligned}
& s_{1}=e^{-\lambda_{11}\left(1-s_{1}\right)} \cdot e^{-\lambda_{12}\left(1-s_{2}\right)}, \\
& s_{2}=e^{-\lambda_{21}\left(1-s_{1}\right)} \cdot e^{-\lambda_{22}\left(1-s_{2}\right)} .
\end{aligned}
$$

Therefore, the invasion probabilities $\pi_{1}$ and $\pi_{2}$ of $A_{1} B_{1}$ and $A_{1} B_{2}$, respectively, as well as the mean invasion probability $\bar{\pi}$ of $A_{1}$, defined in equation (7), can be computed numerically. Below, we refer to this as the (exact) numerical solution.

Remark 6. (i) If $m=0$, then (9b), (6), and (7) show that $\lambda_{12}=0$ and $\bar{\pi}=\pi_{1}$. Therefore, by (23a), $\bar{\pi}$ depends only on $\lambda_{11}$, which is independent of $r$ if $m=0$. Thus, $\bar{\pi}$ is independent of $r$ if $m=0$. The simple intuitive explanation is that in this case allele $B_{1}$ is fixed on the island under our initial equilibrium condition (see eq. 6) so 
that the mutant $A_{1}$ occurs always on the background $B_{1}$, which makes recombination irrelevant in the early phase of invasion.

(ii) If $r=0$, then $\lambda_{12}=\lambda_{21}=0$ and the principal eigenvalue of $\mathbf{M}$ becomes $\lambda=\lambda_{11}=1+(a+e) /(1+b)$. Therefore, $\pi_{1}$ depends only on $\lambda_{11}$, and $\pi_{2}$ depends only on $\lambda_{22}=(1+a) /(1+b)$, which is less than 1 because we assume $a<b$. Therefore, $\pi_{2}=0$ and $\bar{\pi}=q_{B} \pi_{1}$, which depends only on $a+e$ but not on $a$ and $e$ separately. Also this result has a simple intuitive explanation: In the absence of recombination, the mutant cannot invade if it occurs on the 'bad' background $B_{2}$, because on this background its invasion rate is $\lambda_{22}<1$ and without recombination it cannot recombine onto the 'good' background $B_{1}$. Because the invasion rate of $A_{1} B_{1}$ is $\lambda_{11}=1+(a+e) /(1+b)$, the migration rate enters the invasion probability $\bar{\pi}=q_{B} \pi_{1}$ only through the equilibrium frequency of $B_{1}$.

In general, the transcendental equations in (23) cannot be solved analytically. However, we can obtain approximations of the invasion probabilities by assuming a slightly supercritical branching process. For this purpose we assume that the mean matrix $\mathbf{M}=\mathbf{M}(\epsilon)$ depends on a parameter $\epsilon>0$ such that its leading eigenvalue, given in (11), can be written as $\lambda(\epsilon)=1+\rho(\epsilon)$, where $\rho(\epsilon) \rightarrow 0$ as $\epsilon \rightarrow 0$.

Let $u(\epsilon)=\left(u_{1}(\epsilon), u_{2}(\epsilon)\right)$ and $v(\epsilon)=\left(v_{1}(\epsilon), v_{2}(\epsilon)\right)^{T}$ be the positive left and right eigenvectors of $\mathbf{M}(\epsilon)$ corresponding to the leading eigenvalue $\lambda(\epsilon)$. They are normalized such that

$$
\sum_{j=1}^{2} u_{j}(\epsilon)=1=\sum_{i=1}^{2} u_{i}(\epsilon) v_{i}(\epsilon)
$$

holds. For a slightly supercritical branching process, the invasion probabilities $\pi_{i}$ are of order $\epsilon$ and can be expressed as $\pi_{i}=\pi_{i}(\epsilon)+o(\epsilon)$, where

$$
\pi_{i}(\epsilon)=\frac{2(\lambda(\epsilon)-1)}{\beta(\epsilon)} v_{i}(\epsilon)
$$

and $\beta(\epsilon)$ is a complicated expression (Athreya, 1993; Haccou et al., 2005, pp. 126-128). This approximation is valid for general offspring distributions, such that the matrix $\mathbf{M}(\epsilon)$ is primitive for every $\epsilon>0$, i.e., some power is strictly positive. Under the assumption of independent Poisson offspring distributions, we obtain (Appendix A)

$$
\beta(\epsilon)=\lambda(\epsilon)^{2} \sum_{j=1}^{2} u_{j}(\epsilon) v_{j}(\epsilon)^{2} .
$$

Aeschbacher and Bürger (2014, eq. (64) in File S1 of their Supporting Information) 
provided a formula equivalent to

$$
\beta_{\bullet}(\epsilon)=\beta(\epsilon)+\lambda(\epsilon)\left(1-\sum_{j=1}^{2} u_{j}(\epsilon) v_{j}(\epsilon)^{2}\right) .
$$

In general, $\beta_{\bullet}(\epsilon)$ differs from $\beta(\epsilon)$, which is the correct slightly supercritical approximation in the sense of Athreya. In Appendix A we prove that $\beta_{\bullet}(\epsilon) \leq \beta(\epsilon)$ holds always. In addition, we show there that $\beta(\epsilon)=\beta_{\bullet}(\epsilon)$ if $m=0, r=0, m=m^{*}$, or $r=r^{*}$, where in the last two cases $\epsilon=0$ by Remark 3(vi).

Remark 7. We refrain from giving explicit expressions of quantities such as $\mathbf{M}(\epsilon), \beta(\epsilon)$, or $\bar{\pi}(\epsilon)$ because they are very complicated. They could be obtained, for instance, by setting $m=m^{*}-\epsilon$ since we have noted above that $\epsilon=0$ if $m=m^{*}$. In our numerical examples we (almost) always have $m^{*}<0.1$, which would imply $\epsilon<0.1$ because $m>0$. In addition, $\lambda$ is strictly declining as a function of $r$ (File S1, Sect. 6) which assumes its maximum $1+(a+e) /(1+b)$ at $r=0$ (Remark 6(ii)) and decays to the value 1 as $r$ increases to $r^{*}$ (see above). Therefore, the condition of supercriticality will be satisfied if $(a+e) /(1+b)=O(\epsilon)$ or, simpler, if $a=O(\epsilon)$ and $e=O(\epsilon)$. Again, rewriting the relevant expressions in terms of $\epsilon$ makes them very cumbersome. Therefore, in the sequel, we indicate the dependence of $\epsilon$ only to signify that we use the assumption of slight supercriticality.

\subsection{Properties of the mean invasion probability $\bar{\pi}$ and its ap- proximations}

Now we apply the above approximations to our mean matrix (9b) to investigate the properties of $\bar{\pi}$ in dependence on the parameters $a, b, e, r$, and $m$. The resulting explicit expressions for the leading terms $\pi_{i}(\epsilon)$ and $\bar{\pi}(\epsilon)=q_{B} \pi_{1}(\epsilon)+\left(1-q_{B}\right) \pi_{2}(\epsilon)$ of the invasion probabilities $\pi_{i}$ and the mean invasion probability $\bar{\pi}$, obtained by substituting the explicit expression $\beta(\epsilon)$ from (26) into (25), are complicated and not very informative (see, e.g., (11) for the principal eigenvalue $\lambda$ ). They are derived and presented in File S3, Sect. 2. Below, we present simple explicit approximations only for special cases.

In the sequel, we write $\bar{\pi}_{\bullet}(\epsilon)$ for approximation of $\bar{\pi}$ obtained by substituting the explicit expression for $\beta_{\bullet}(\epsilon)$ in (27) into (25). The inequality $\beta_{\bullet}(\epsilon) \leq \beta(\epsilon)$ implies $\bar{\pi}_{\bullet}(\epsilon) \geq \bar{\pi}(\epsilon)$. Despite the fact that the difference between $\beta(\epsilon)$ and $\beta \bullet(\epsilon)$ can be of order $\epsilon$, it turns out that for a relatively wide range of parameters, including small recombination rates, $\bar{\pi}_{\bullet}(\epsilon)$ provides a more accurate approximation of $\bar{\pi}$ than $\bar{\pi}(\epsilon)$ (Figure 
A

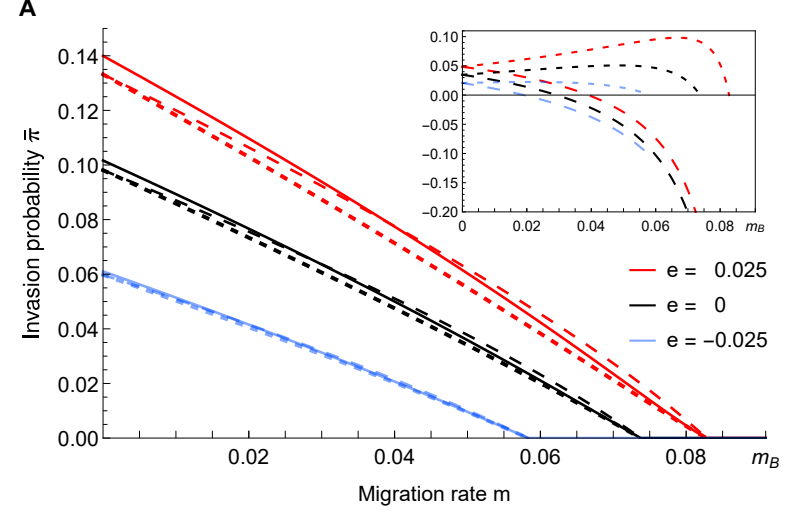

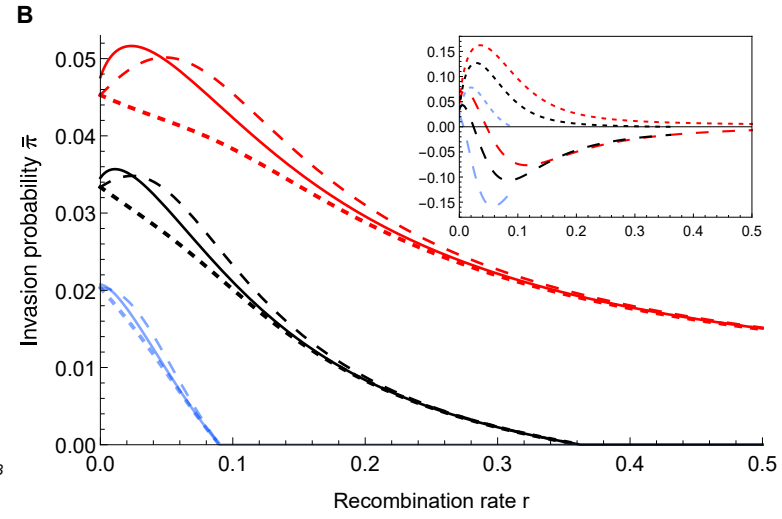

Figure 2: Dependence of the mean invasion probability $\bar{\pi}$ on the migration rate (A) and the recombination rate (B) for three different values of epistasis. The solid curves show $\bar{\pi}$, where the numerical solution $\left(\pi_{1}, \pi_{2}\right)$ of the exact equations $(23)$ has been substituted into (7). The dotted curves show the approximation $\bar{\pi}(\epsilon)$, and the dashed curves show $\bar{\pi}_{\bullet}(\epsilon)$ (File S3.2). The line color legend in A applies to both panels. The insets show the relative error of the approximations with respect to the numerical solution of (23). The dashing of the curves in the inset corresponds to the dashing of the approximations in the main figure. In both panels we have $a=0.06$ and $b=0.1$. In panel $\mathrm{A}, r=0.1$ and in panel $\mathrm{B}, m=0.06$.

2). In particular, it better reflects certain qualitative features of the true (numerically computed) $\bar{\pi}$ as a function of $r$.

In the absence of migration, we obtain the approximation

$$
\bar{\pi}(\epsilon)=\bar{\pi}_{\bullet}(\epsilon)=\frac{2(1+b)(a+e)}{(1+b+a+e)^{2}} \quad(\text { at } m=0),
$$

and in the absence of recombination, we obtain

$$
\bar{\pi}(\epsilon)=\bar{\pi}_{\bullet}(\epsilon)=\frac{2(1+b)(a+e)}{(1+b+a+e)^{2}}\left(1-\frac{m(1+b)}{b}\right) \quad(\text { at } r=0) .
$$

Both are readily derived from the respective first-order approximations of $\pi_{i}(\epsilon)$ (see eqs. (B.1) and (B.4) in Appendix B and File S3, Sects. 2, 6). In confirmation of Remark $6(\mathrm{i})$, the approximation (28) is independent of $r$ in the absence of migration.

The first-order approximation $\bar{\pi}(\epsilon)$ near $m=0$ is given by eq. (B.5a) in Appendix $\mathrm{B}$ and is decreasing in $m$. We conjecture that $\bar{\pi}$ is generally decreasing in $m$, because this is supported by all our numerical results. However, as is clearly visible in Fig. 2 and shown analytically below, $\bar{\pi}$ and its approximations are not necessarily decreasing in $r$.

Figure 2 demonstrates that both approximations, $\bar{\pi}(\epsilon)$ and $\bar{\pi}_{\bullet}(\epsilon)$, are quite accurate. This is consistent with the fact that they are identical if $m=0, m=m^{*}, r=0$, and 
$r=r^{*}$. Whereas $\bar{\pi}(\epsilon)$ appears to be always smaller than the exact value of $\bar{\pi}, \bar{\pi}_{\bullet}(\epsilon)$ can be smaller or greater. The parameters used in Fig. 2A imply $e_{r} \approx 0.06$. Because $e<e_{r}$ for all three choices of $e$, all curves decay to 0 in the interval $\left(0, m_{B}\right)$ by Proposition 1. The critical values above which invasion becomes impossible are $m^{*} \approx 0.083$ for the red curves, $m^{*} \approx 0.074$ for the black curves, and $m^{*} \approx 0.058$ for the blue curves. In Fig. $2 \mathrm{~B}$ we have $e_{m} \approx 0.004$. From Proposition 2 we know that epistasis is sufficiently strong to enable invasion for every recombination probability (including free recombination) if and only if $e>e_{m}$. This is the case for the red curves, where $e=0.025$. In the absence of epistasis (black curves), we have $\bar{\pi}>0$ only if $r<r^{*} \approx 0.36$, and for $e=-0.025$ (blue curves), we have $\bar{\pi}>0$ only if $r<r^{*} \approx 0.09$.

Figure 2 shows that increasing epistasis (larger $e$ ) increases the mean invasion probability of $A_{1}$, and all our numerical results confirm this. A general proof seems difficult, but at least the principal eigenvalue $\lambda$ is increasing in $e$ by Remark 3 . With the scaling of epistasis as in (1), this is not surprising because increasing $e$ raises the fitness of $A_{1} B_{1}$. Whereas for tightly linked mutants $A_{1}$ positive epistasis has only a quantitative effect by increasing the invasion probability, for loosely linked or unlinked mutants it may have a qualitative effect by enabling invasion.

The weak-forces approximations of the invasion probabilities are simpler than those used above, but still quite complicated. They are provided in Appendix C.

For weak migration and if $A_{1}$ alleles have a small effect and are loosely linked to the existing polymorphism, a simple and useful approximation for the invasion probability is obtained. Indeed, if we assume $a, m, e \ll r, b$ and use either $\bar{\pi}(\epsilon)$ or $\bar{\pi}_{\bullet}(\epsilon)$, we find to leading order in $a, m$, and $e$ :

$$
\bar{\pi}(\epsilon) \approx \pi_{1}(\epsilon) \approx \bar{\pi}_{w}=2 \frac{(a+e)(b+r)-(1+b) m r}{(1+b)(b+r)} .
$$

The reason why $\bar{\pi}(\epsilon) \approx \pi_{1}(\epsilon)$ is that if $a, m \ll b$, the frequency of $B_{1}$ at the equilibrium $E_{B}$ is close to one. In addition, the invasion probability $\pi_{2}$ of $A_{1}$ on the deleterious background $B_{2}$ is always smaller than $\pi_{1}$. Therefore, the contribution of the term $\left(1-q_{B}\right) \pi_{2}(\epsilon)$ in $(7)$ to $\bar{\pi}(\epsilon)$ is negligible in this case.

The approximation $\bar{\pi}_{w}$ in (30) is increasing in $a$ and $e$, and decreasing in $m$ and $r$. It shares these properties with the invasion condition (Remark 3) because $\bar{\pi}(\epsilon)=$ $2(\lambda(\epsilon)-1)$ to first order in $a, m, e$ if $a, m, e \ll r, b$. If $e=0$, the approximation (30) simplifies to equation (4) in Yeaman et al. (2016).

From (30), we immediately derive the following approximation for the effect of epis- 
tasis on the invasion probability:

$$
\bar{\pi}_{w}(e)-\bar{\pi}_{w}(0)=\frac{2 e}{1+b} .
$$

Here, $\bar{\pi}_{w}(e)$ indicates the dependence on $e$. In fact, $\frac{2 e}{1+b}$ seems to be an upper bound for the increase of the invasion probability caused by epistasis; it is most accurate for very small $m$ (Fig. 3).

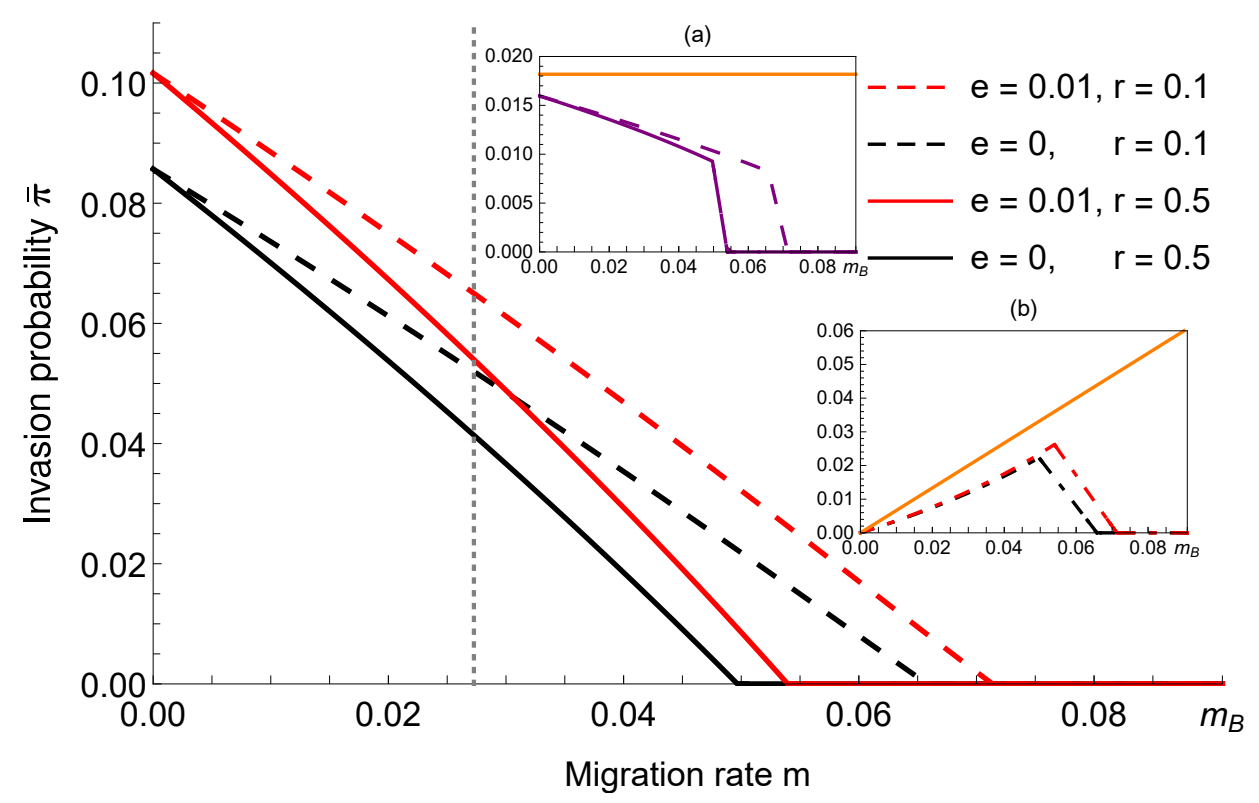

Figure 3: Comparison of the effects of epistasis with those of linkage on the mean invasion probability $\bar{\pi}$, which is shown for the four possible combinations of the two values of $e$ and the two values of $r$ shown in the legend. The parameters $a=0.05$ and $b=0.1$ are kept constant. The figure not only confirms that positive epistasis and linkage increase $\bar{\pi}$ relative to no epistasis and no linkage, but in particular shows that the red solid and the black dashed curves intersect. Therefore, epistasis may be more efficient than linkage in increasing $\bar{\pi}$ if migration is weak, whereas tighter linkage may be more efficient if migration is strong. The vertical dotted line shows the analytical prediction, $\hat{m}_{w}$ in (35), for this intersection point. Curves of the same color, i.e., with the same $e$, coincide at $m=0$ by Remark 6(i) and assume the approximate values (28). Inset (a) shows the difference between the two curves of the same line style (dashed or solid), i.e., the effect of epistasis on $\bar{\pi}$. Inset (b) shows the difference between the two curves of the same line color, i.e., the effect of linkage on $\bar{\pi}$. The orange curve in each inset represents the analytical prediction of the respective difference as given in (31) and (32), respectively.

Similarly, the effect of linked relative to unlinked loci can be estimated by

$$
\bar{\pi}_{w}(r)-\bar{\pi}_{w}\left(\frac{1}{2}\right)=\frac{2 m b(1-2 r)}{(1+2 b)(b+r)},
$$


whereas

$$
\bar{\pi}_{w}\left(m_{1}\right)-\bar{\pi}_{w}\left(m_{2}\right)=\frac{2 r\left(m_{2}-m_{1}\right)}{b+r}
$$

yields the effect of differences in the migration rate. The expressions (31), (32), and (33) give crude but simple approximations for the effects of varying a single parameter (see the orange curves in the insets of Figs. 3 and 4).

Finally, the solution of the equation

$$
\bar{\pi}_{w}(e=0)-\bar{\pi}_{w}\left(r=\frac{1}{2}\right)=0,
$$

in terms of $m$ is

$$
\hat{m}_{w}=\frac{(1+2 b)(b+r) e}{b(1+b)(1-2 r)} .
$$

This is the value of $m$, for which the mean invasion probability at an unlinked epistatic locus is the same as at a linked nonepistatic locus (see Fig. 3).

In Fig. 2, we have already seen that increasing epistasis and decreasing recombination tend to increase the mean invasion probability. Figure 3 also shows this effect, but in addition it shows the following remarkable effect. If migration is weak, then positive epistasis between unlinked loci is more efficient than stronger linkage between non-epistatic loci in boosting the mean invasion probability, whereas if migration is strong, linkage is more efficient (because the red solid curve and the black dashed curve intersect). A reasonably accurate estimate of the critical migration rate at which these curves intersect is given in (35) and is shown as a vertical dashed line in the figure.

In Fig. 4, the mean invasion probability $\bar{\pi}$ is displayed as a function of $r$ for different combinations of $a, e$, and $m$. In particular, $a$ and $e$ are individually varied such that $a+e=$ constant. Therefore, the fitness of $A_{1} B_{1}$ is fixed for all shown combinations, and only the fitness of haplotype $A_{1} B_{2}$ varies with $a$. The reasoning behind this choice is that, instead of (1), the epistasis parameter $e$ could be defined by

$$
W=\left(\begin{array}{cc}
B_{1} & B_{2} \\
1+a^{\prime}+b & 1+a^{\prime}-e \\
1+b & 1
\end{array}\right) \begin{gathered}
A_{1} \\
A_{2}
\end{gathered}
$$

where $a^{\prime}=a+e$. Which scaling is more appropriate may depend on the situation that one intends to model and is discussed below. In light of this parametrization, Remark 6(ii) can be reformulated such that the value of $\bar{\pi}$ at $r=0$ is determined only by the parameter $a^{\prime}$, and not by $e$. For $r>0, \bar{\pi}$ depends on $a^{\prime}$ and on $e$, as is shown by the divergence among curves of the same dashing style. This is also the case for the first-order approximation of $\bar{\pi}$ (eq. (B.2) in Appendix B). 


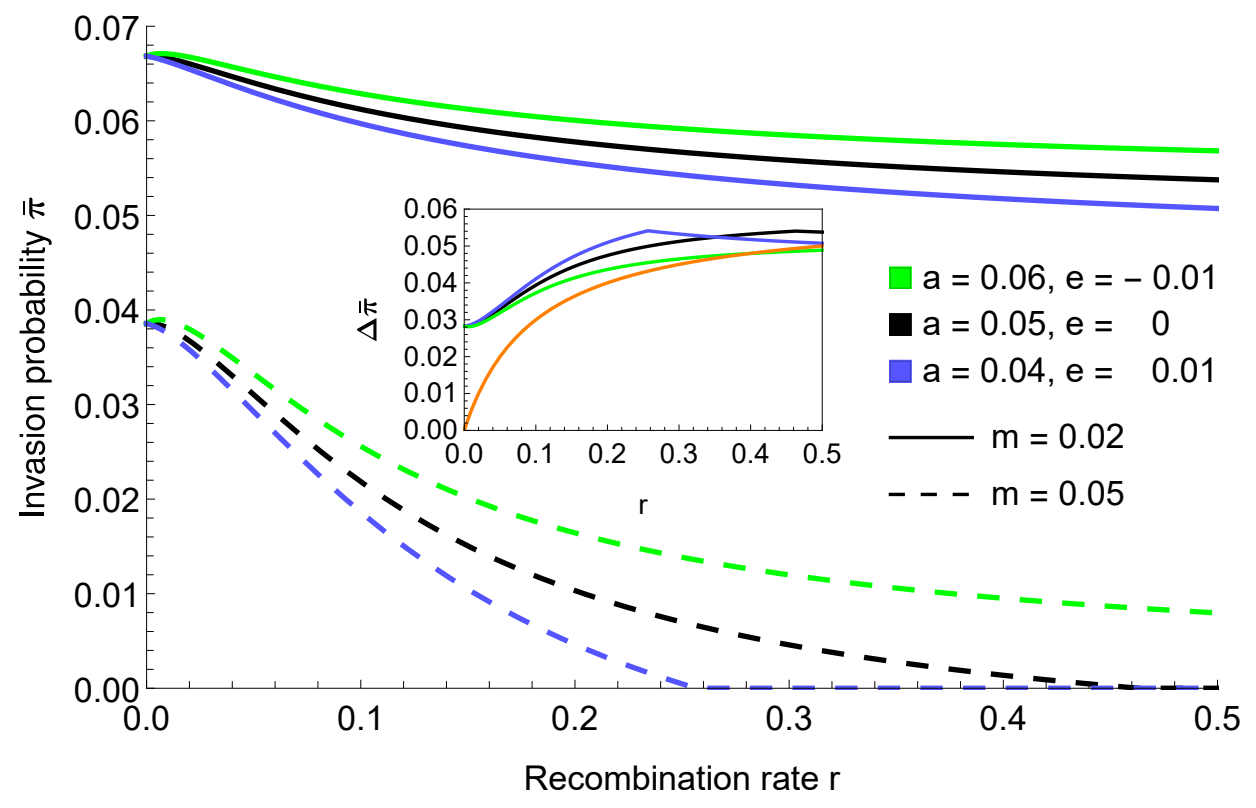

Figure 4: The mean invasion probability as a function of the recombination rate $r$ under an alternative parameterization of epistasis. Here, $b=0.1$ is constant as in the other figures, but $a$ and $e$ are varied such that the fitness of the best genotype, $A_{1} B_{1}$, is held constant at $1+a^{\prime}+b=1+a+e+b=1.15$. Hence, the fitness of $A_{1} B_{2}$ varies. The inset shows the difference between curves of the same color, i.e., the effect of migration if everything else stays constant. The orange curve represents the analytical approximation (33). All other curves show the exact numerical solution obtained from (23).

Figure 4 shows that the rate of decline of $\bar{\pi}$ with increasing $r$ and constant $a^{\prime}$ is determined by the value of $e$. A small positive, or even negative value, of $e$ entails a slower decay of $\bar{\pi}$, because increasing $e$ reduces the fitness $1+a^{\prime}-e$ of the haplotype $A_{1} B_{2}$.

The difference of the invasion probabilities for different migration rates (difference between curves of the same color) is shown in the inset. The analytical approximation (33) (orange curve) is quite accurate for strong recombination, but fails for low recombination. This is not unexpected because the approximation (30), from which (33) is derived, assumes large $r$.

Finally, it seems worth noting that $\bar{\pi}$ is more sensitive to changes in the recombination rate $r$ if $m$ is relatively large compared to $a$. In particular, for given selection parameters, the decay of $\bar{\pi}$ as a function of $r$ is faster if $m$ is larger (compare the dashed with the solid curves in Fig. 4). 


\subsection{Non-zero optimal recombination rate}

Figure 2B demonstrates that for small $r, \bar{\pi}$ can increase and be maximized at a non-zero recombination rate, referred to as the optimal recombination rate. The red and black curves in Fig. 2B provide examples, in which the (exact) mean invasion probability $\bar{\pi}$ (solid curves) and the approximation $\bar{\pi}_{\bullet}(\epsilon)$ (dashed curves) have a non-zero optimal recombination rate, whereas $\bar{\pi}(\epsilon)$ (dotted curves) has its maximum at $r=0$.

We find an estimate of the parameter region in which the optimal recombination rate is greater than zero by computing a critical value $a^{*}$ such that $\partial \bar{\pi}(\epsilon) / \partial r<0$ at $r=0$ if $a<a^{*}$, and $\partial \bar{\pi}(\epsilon) / \partial r>0$ at $r=0$ if $a>a^{*}$. In the latter case, $\bar{\pi}(\epsilon)$ has a local maximum at some $r>0$ (potentially, at $r=\frac{1}{2}$, see Fig. S1). From the first-order approximation $\bar{\pi}(\epsilon)$ in (B.2), we derive immediately

$$
a^{*}=-\frac{1+2 b+e(2+b)-\sqrt{1+8 b+4 e+6 b(2 b+e)+b^{2}\left(4 b+e^{2}\right)}}{2(1+b)}>0 .
$$

Analogously, we obtain from $\bar{\pi}_{\bullet}(\epsilon)$ in (B.3)

$$
a_{\bullet}^{*}=-\frac{2+3 b+e(2+b)-\sqrt{4+16 b+4 e+b(4 e+17 b)+b^{2}\left(4 b-2 e+e^{2}\right)}}{2(1+b)}>0 .
$$

For the parameters in Fig. 2B, where $a=0.06$, the critical values of $a$ are as follows.

- If $e=0.025$ (red curves), then $a^{*} \approx 0.080$ and $a_{\bullet}^{*} \approx 0.033$.

- If $e=0$ (black curves), then $a^{*} \approx 0.085$ and $a_{\bullet}^{*} \approx 0.047$.

- If $e=-0.025$ (blue curves), then $a^{*} \approx 0.090, a_{\bullet}^{*} \approx 0.0602$.

In accordance with the observation in Fig. $2 \mathrm{~B}$ that $\bar{\pi}_{\bullet}(\epsilon)$ exhibits an optimal recombination rate if the true $\bar{\pi}$ does (as for the red and black curves), we see that $a_{\bullet}^{*}<a<a^{*}$ in these two cases. In the third case, $a<a_{\bullet}^{*}<a^{*}$ and, indeed, the corresponding blue curves are monotonically decreasing.

By assuming weak evolutionary forces and applying the corresponding limit (Sect. 3.2) to $a^{*}$ (or working with (C.3)), we obtain

$$
a^{*} \approx \tilde{a}^{*}=b
$$

Therefore, with weak evolutionary forces and because $a<b, \bar{\pi}(\epsilon)$ decreases with increasing recombination near $r=0$. In contrast, the weak-forces approximation applied to $a_{\bullet}^{*}$ yields

$$
a_{\bullet}^{*} \approx \tilde{a}_{\bullet}^{*}=\frac{b-e}{2}
$$


such that a nonzero optimal recombination rate is predicted by this approximation if $a>\frac{b-e}{2}$ or, equivalently, if $b-a<a+e$. In terms of the fitness matrix (1) the latter can be written as $w_{11}-w_{21}>w_{21}-w_{12}$. This means the fitness benefit of having $A_{1}$ instead of $A_{2}$ on the $B_{1}$ background is greater than that of having the genotype $A_{2} B_{1}$ instead of $A_{1} B_{2}$. Indeed, this inequality is satisfied for the red and black dashed curves in Fig. 2B, but not for the blue curves. Hence, (40) provides a rough, but simple, estimate of when $\bar{\pi}$ exhibits a non-zero optimal recombination rate, whereas (39) never predicts this phenomenon. In particular, (40) shows that increasing epistasis increases the range of values $a$ for which $\bar{\pi}(\epsilon)$ is maximized at a positive recombination rate. This holds more generally for $a^{*}$ and $a_{\bullet}^{*}$, which both decrease with increasing $e$ (see File S3, Sect. 4).

\section{$5 \quad$ Averaged invasion probabilities}

Efficient local adaptation of the island population will require the successful invasion, or establishment, of several, if not many, weakly beneficial mutants. They can occur at an arbitrary physical distance to the already established polymorphism, and neither their additive nor their epistatic fitness effects will be known a priori (and often also not a posteriori). Therefore, we investigate various scenarios in which the recombination rate between loci $A$ and $B$ and the effects $a$ and $e$ of the new mutants are drawn from appropriate distributions. To obtain expected invasion probabilities of the new mutant $A_{1}$, we need to integrate the mean invasion probability $\bar{\pi}$ with respect to the chosen distributions of the parameters $r, a$, and $e$.

For the recombination rate between $A$ and $B$ we follow Yeaman et al. (2016) and assume a uniform distribution of $r \in\left[0, \frac{1}{2}\right]$; we denote the corresponding probability density by $u(r)$. For the distribution of additive fitness effects $a$, we assume an exponential distribution with mean $\bar{a}$, which we denote by

$$
f(a)=\frac{1}{\bar{a}} e^{-a / \bar{a}}
$$

For the distribution of epistatic effects, we consider two cases. In the first, we follow Martin et al. (2007), who deduced an epistasis distribution from an extended version of Fisher's geometric model and fitted it to two empirical data sets from haploid organisms. Their epistasis distribution is a normal distribution with mean 0 and variance twice the variance of the additive fitness effect of the new mutation (thus, independent of the 'ancestral strain'), i.e.,

$$
\mathrm{E}[h]=0 \text { and } \operatorname{Var}[h]=2 \bar{a}^{2},
$$


where $h$ denotes the probability density of $e$. Defined in this way, $h(e)$ is a distribution of epistatic interactions between random mutations (i.e., beneficial or deleterious), whereas in our model both mutations $\left(A_{2} \rightarrow A_{1}\right.$ and $\left.B_{2} \rightarrow B_{1}\right)$ are beneficial.

Building on the work by Martin et al. (2007), Blanquart et al. (2014) provided results for the mean and variance of a distribution of genetic interactions between beneficial mutations. Denoting the density of epistatic effects between beneficial mutations by $h_{+}$, we conclude from their equation (S13) that $h_{+}$is a normal distribution with mean and variance given by

$$
\mathrm{E}\left[h_{+}\right]=-\pi \bar{a} \text { and } \operatorname{Var}\left[h_{+}\right]=\left(16-\pi^{2}\right) \bar{a}^{2},
$$

which implies that $10.2 \%$ of all mutations have a positive epistatic effect and $\operatorname{Var}\left[h_{+}\right] \approx$ $3.1 \operatorname{Var}[h]$.

Because analytical evaluation of the expectations of the approximate invasion probability $\bar{\pi}(\epsilon)$ with respect to the distributions of $r, a$, or $e$ seems unfeasible, we present only results from numerical integration of the exact, numerically determined $\bar{\pi}$. Our main focus is on the role of linkage and epistasis.

We use notation such as

$$
\begin{gathered}
\bar{\pi}_{f}=\int_{0}^{\infty} \bar{\pi}(a) f(a) d a, \quad \bar{\pi}_{f, u}=\int_{0}^{1 / 2} \int_{0}^{\infty} \bar{\pi}(a, r) f(a) d a u(r) d r \\
\bar{\pi}_{f, u, h}=\int_{-\infty}^{\infty} \int_{0}^{1 / 2} \int_{0}^{\infty} \bar{\pi}(a, r, e) f(a) d a u(r) d r h(e) d e
\end{gathered}
$$

for invasion probabilities averaged over the indicated distributions. To emphasize that a particular value is fixed, e.g., $e=0$ or $r=\frac{1}{2}$, we indicate this by additional subscripts, e.g., by $\bar{\pi}_{f, e=0}$ or $\bar{\pi}_{f, h, r=1 / 2}$, respectively. Since the distributions are independent, a different order of the subscripts does not change the result.

To approximate, for instance, the integral $\bar{\pi}_{f, u}$, we define a sufficiently fine grid $(a, r)$. At each grid point the numerical value of the integrand is computed. The resulting table of values is approximated by a continuous (bivariate) function with the built-in Mathematica method Interpolation. This continuous function is then integrated numerically over $a$ and $r$ (again using Mathematica) to obtain an approximation for $\bar{\pi}_{f, u}$.

Figure 5A shows that linkage affects the averaged invasion probability in essentially the same way for all three values $e=-0.005,0,0.005$ (compare the shapes of the curves of the same line style but of different color). The averaged invasion probability is always declining in $m$, and for large $r$ this decline is much steeper than for small 
A

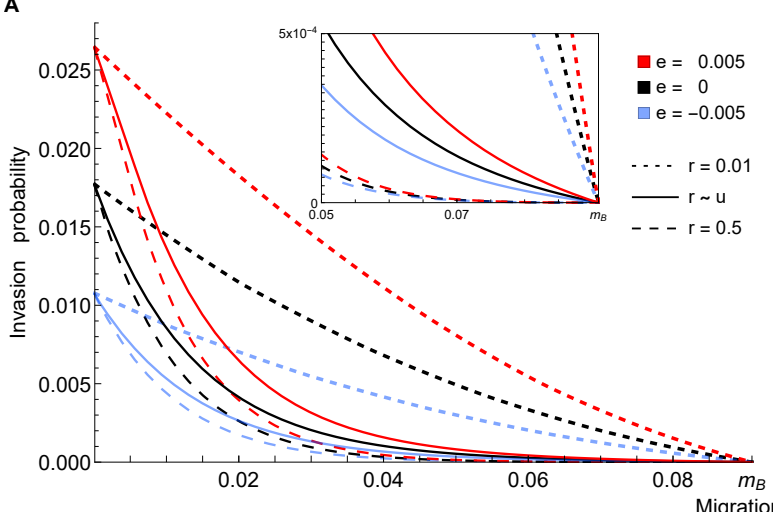

B

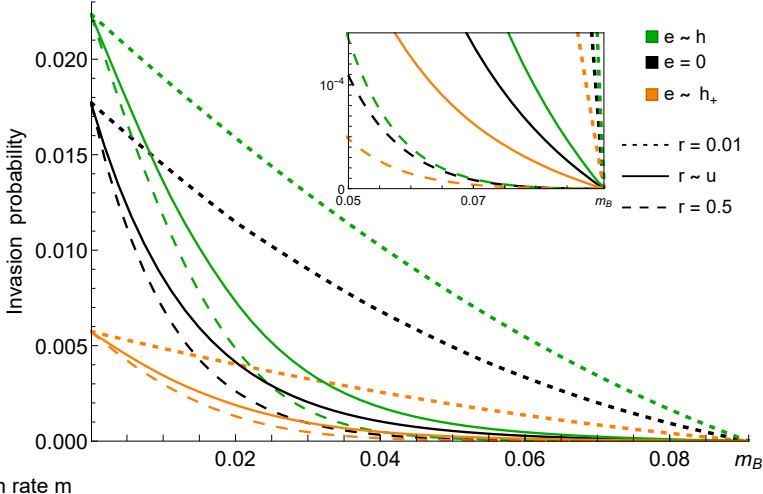

Figure 5: Averaged invasion probabilities of new mutations as functions of $m$. In all cases, the invasion probability is averaged with respect to $f(a)$ with mean $\bar{a}=0.01$. The parameter $b$ is fixed at $b=0.1$, so that the maximum possible migration rate is $m_{B} \approx 0.091$. (A) For each of the (fixed) epistatic values $e=0.005,0,-0.005$, the averged invasion probabilities of $A_{1}$ under free recombination $\left(\bar{\pi}_{f, r=1 / 2}\right)$ and under tight linkage $\left(\bar{\pi}_{f, r=0.01}\right)$ are compared with that under a uniform distribution $\left(\bar{\pi}_{f, u}\right)$, indicated in the legend by $r \sim u$. The inset shows the approach to zero for $m$ close to $m_{B}$. (B) is analogous to (A) except that $e$ is drawn from the distributions $h$ (indicated by $e \sim h$, green $)$ or $h_{+}\left(e \sim h_{+}\right.$, orange $)$. Here, $\mathrm{E}[h]=0, \operatorname{Var}[h]=2 \bar{a}^{2}=0.0002$, and $\mathrm{E}\left[h_{+}\right]=-\pi \bar{a} \approx-0.031, \operatorname{Var}\left[h_{+}\right] \approx 0.00061$, by (42) and (43), respectively. The black curves in $\mathrm{A}$ and $\mathrm{B}$ (for which $e=0$ ) are identical.

$r$. Indeed, when $m$ gets close to $m_{B}$, invasion probabilities group together that are computed with the same strength of linkage (see inset), whereas for small $m$ invasion probabilities based on the same $e$ are similar, and identical if $m=0$ (Remark 6(i)). Because $a$ is drawn from the exponential distribution $f$, mutants of large effect can occur and invasion becomes possible for every $m \leq m_{B}$. However, for large $m$ the invasion probability may be negligibly small, especially if $r$ is large (see inset). Thus, in concordance with previous results (e.g., Fig. 1B and Fig. 4), this figure confirms that tight linkage becomes essential for facilitating, or even enabling, invasion if migration rates are high.

Figure 5B demonstrates the consequences of drawing epistatic effects from either the distribution $h$ (green curves) or $h_{+}$(orange curves). In the first case, where $\mathrm{E}[h]=0$, the invasion probability close to $m=0$ is higher than if $e=0$, regardless of the degree of linkage (cf. Remark 6). In the second case, in which epistatic effects are negative on average, i.e., $\mathrm{E}\left[h_{+}\right]<0$, the invasion probability (orange curves) for small migration rates is reduced substantially compared to both other scenarios.

Notably, in Figs. 5A and 5B, curves of different color and dashing style can cross. 
For instance in B, the short-dashed black curve is above the solid and the long-dashed green curve unless $m$ is very small. Thus, tight linkage provides a greater advantage for invasion than a higher fitness $(1+b+a+e)$ of the double mutant $A_{1} B_{1}$ caused by positive epistasis. For small $m$, the higher fitness provides the greater advantage. Similarly, above a critical migration rate, the invasion probability of tightly linked mutations with, on average, negative epistasis (short-dashed orange curve) is higher than that of unlinked or loosely linked mutations with no epistasis (long-dashed and solid black curves), and even higher than that with, on average, positive epistasis (long-dashed and solid green curves). We observed this effect already in Fig. 3 for fixed values of $e$ and $r$, where we discussed it briefly. In particular, (35) provides a reasonably accurate approximation for the critical value $m$ at which the advantages of higher epistasis and tighter linkage balance.

The effects of linkage and epistasis on the invasion probability are further highlighted in Fig. S3, which is based on the data and graphs in Fig. 5, and in Fig. S4. Both demonstrate that the epistasis distribution $h$, which has mean 0 , facilitates invasion of $A_{1}$ compared to absence of epistasis, whereas the epistasis distribution $h_{+}$, which has a negative mean, impedes it. In addition, Fig. S3B shows that if migration is weak, invasion at unlinked loci contributes more to the average invasion probability than invasion at linked loci, whereas the contribution of linked loci matters most if migration is strong.

\section{Average effects of successfully invading mutants}

If the mutational effects, additive or epistatic, are drawn from distributions, mutants with larger effects will have an elevated invasion probability. However, mutants with much larger effects than the mean will also have a substantially reduced probability of occurrence. Here, we explore the average effects of successfully invading mutants numerically; given the complexity of the approximations for $\bar{\pi}$ an analytical treatment seems out of reach. In view of the applications in the next section, we study the average effects as functions of the recombination rate between the two loci.

Each mutant has an additive effect $a$, drawn from the exponential distribution $f$ in (41), and an epistatic effect $e$ drawn from either the normal distribution $h$ in (42) or the normal distribution $h_{+}$in (43). For given parameters $b, m, r$, and $\bar{a}$ (the mean of $f$ ) and following the previous notation, we denote the invasion probabilities averaged 
over $f$ and $h$ or over $f$ and $h_{+}$by

$$
\bar{\pi}_{f, h}=\int_{-\infty}^{\infty} \int_{0}^{\infty} \bar{\pi}(a, e) f(a) h(e) d a d e,
$$

or

$$
\bar{\pi}_{f, h_{+}}=\int_{-\infty}^{\infty} \int_{0}^{\infty} \bar{\pi}(a, e) f(a) h_{+}(e) d a d e
$$

respectively. Then the average effects $(a, e)$ of the successfully invading mutations, i.e., conditioned on invasion, are given by

$$
\begin{aligned}
\bar{a}_{s} & =\int_{-\infty}^{\infty} \int_{0}^{\infty} a \bar{\pi}(a, e) f(a) h(e) d a d e / \bar{\pi}_{f, h}, \\
\bar{e}_{s} & =\int_{-\infty}^{\infty} \int_{0}^{\infty} e \bar{\pi}(a, e) f(a) h(e) d a d e / \bar{\pi}_{f, h}, \\
\bar{a}_{+, s} & =\int_{-\infty}^{\infty} \int_{0}^{\infty} a \bar{\pi}(a, e) f(a) h_{+}(e) d a d e / \bar{\pi}_{f, h_{+}}, \\
\bar{e}_{+, s} & =\int_{-\infty}^{\infty} \int_{0}^{\infty} e \bar{\pi}(a, e) f(a) h_{+}(e) d a d e / \bar{\pi}_{f, h_{+}} .
\end{aligned}
$$

Figure 6 displays these mutational effects for a variety of migration rates as functions of the recombination rate. As is expected intuitively on the basis of the above developed theory, the average additive effects $\left(\bar{a}_{s}\right.$ and $\left.\bar{a}_{+, s}\right)$ as well as the average total effects $\left(\bar{a}_{s}+\bar{e}_{s}\right.$ and $\left.\bar{a}_{+, s}+\bar{e}_{+, s}\right)$, which are expressed in the island genotype $A_{1} B_{1}$, always increase with increasing migration rate and with increasing recombination rate. This is because the minimal effects of $a$ and $e$ necessary for invasion increase with increasing $m$ or $r$, as follows from Remark 3(ii) or 3(iii), respectively. However, the corresponding invasion probabilities $\left(\bar{\pi}_{f, h}\right.$ and $\left.\bar{\pi}_{f, h_{+}}\right)$decline (often rapidly) with increasing $m$ or $r$ (Fig. S4). Thus, the waiting time until a mutation becomes established at a large recombinational distance from the already polymorphic locus may be very long. If it becomes established, then it likely is a mutation of large effect. 

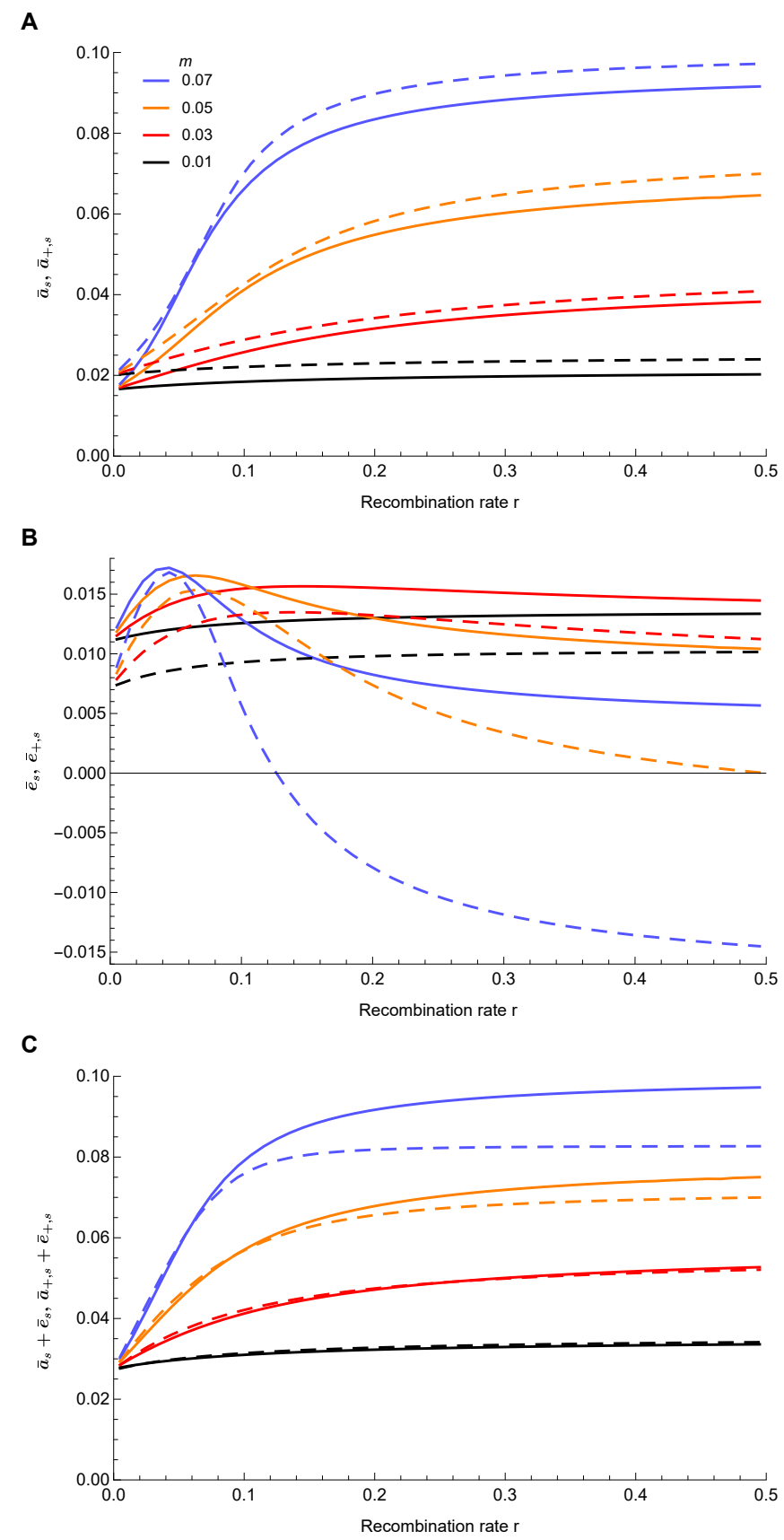

Figure 6: Average additive and epistatic effects of successfully invading mutants as functions of the recombination rate $r$. Additive effects are drawn from an exponential distribution with mean $\bar{a}=0.01$, epistatic effects from $h$ in (42) or $h_{+}$in (43). Panel A shows the resulting average additive effects $\bar{a}_{s}$ (solid) and $\bar{a}_{+, s}$ (dashed) for the indicated migration rates. For unlinked, nonepistatic mutations $\left(r=\frac{1}{2}, e=0\right)$ the minimum effects required for invasion are $a \approx 0.0093$ (if $m=0.01$ ), $a \approx 0.0291$ (if $m=0.03$ ), $a \approx 0.0504$ (if $m=0.05$ ), and $a \approx 0.0735$ (if $m=0.07$ ); the values $\bar{a}_{s}$ and $\bar{a}_{+, s}$ at $r=\frac{1}{2}$ lie well above them. Panel B shows the corresponding average epistatic effects $\bar{e}_{s}$ (solid) and $\bar{e}_{+, s}$ (dashed). Panel $\mathrm{C}$ shows the (total) average effects of $A_{1}$ in the island genotype $A_{1} B_{1}$, i.e., $\bar{a}_{s}+\bar{e}_{s}$ (solid) and $\bar{a}_{+, s}+\bar{e}_{+, s}$ (dashed). In all cases $b=0.1$. 
Panel A shows that $\bar{a}_{+, s} \geq \bar{a}_{s}$ holds always. The reason is that the distribution $h_{+}$ has a negative mean, whereas $h$ has mean zero. Therefore, as panel B shows, $\bar{e}_{+, s} \leq \bar{e}_{s}$ holds and, for the largest migration rate $(m=0.07) \bar{e}_{+, s}$ can even be negative. (Recall from (43) that about $10 \%$ of the mutants drawn from $h_{+}$have a positive effect.) Because a larger total effect $(a+e)$ facilitates invasion, $\bar{a}_{+, s}$ is larger than $\bar{a}_{s}$. As panel $\mathrm{C}$ shows, the average total effects $\bar{a}_{+, s}+\bar{e}_{+, s}$ and $\bar{a}_{s}+\bar{e}_{s}$ are almost identical for the two smaller migration rates, but differ considerably for the largest migration rate; then $\bar{a}_{+, s}+\bar{e}_{+, s}$ is well below $\bar{a}_{s}+\bar{e}_{s}$ if $r \gtrsim 0.12$, because then $\bar{e}_{+, s}<0$ (see panel $\mathrm{B}$ ).

It is also of some interest to compare these average values with the average values of successful mutations that are not epistatic, i.e., if $a$ is drawn from $f$ and $e=0$. For the migration rates $m \leq 0.05$ in Fig. 6 , we obtain the following order, valid for every $r$ :

$$
\bar{a}_{s}<\bar{a}_{+, s}<\bar{a}_{s, e=0}<\bar{a}_{+, s}+\bar{e}_{+, s}<\bar{a}_{s}+\bar{e}_{s}
$$

The first and the last inequality are clearly visible in Fig. 6. If $m=0.7$, then $\bar{a}_{s}<$ $\bar{a}_{s, e=0}<\bar{a}_{s}+\bar{e}_{s}$ still holds, but the other inequalities are violated if $r \gtrsim 0.12$ (results for $\bar{a}_{s, e=0}$ are not displayed).

\section{Approximate size of a genomic island}

Yeaman et al. (2016) discussed several categories of explanation for the occurrence or maintenance of genomic islands of elevated divergence (measured by $F_{S T}$ ) between a pair of parapatric populations. A particularly likely explanation is that linkage of locally beneficial de novo mutations to an already established selection-migration polymorphism facilitates successful invasion of such mutations in the face of maladaptive gene flow. Because linkage depends strongly on physical distance, invasions should be successful predominantly locally around the already polymorphic site and thus lead to genomic islands of divergence.

Similar to the quantity $C_{95}$, which was introduced by Yeaman et al. (2016), we investigate the quantities $C_{50}$ and $C_{90}$, the $50 \%$ and $90 \%$ window sizes. These are the smallest neighborhoods of the polymorphic site in which $50 \%$ and $90 \%$, respectively, of all new mutations became established. Thus, $C_{50}$ is the value of $r$ required to contain $50 \%$ of the probability density of $\bar{\pi}$ as a function or $r$. Formally, $C_{50}$ is defined such that $\int_{0}^{C_{50}} \bar{\pi}(r) d r=0.5 \int_{0}^{1 / 2} \bar{\pi}(r) d r$ holds, and analogously for $C_{90}$.

A small value of $C_{50}\left(C_{90}\right)$ corresponds to a locally restricted genomic region in which the mutations profit from their linkage to the polymorphic site. A high value of $C_{50}$ $\left(C_{90}\right)$ indicates that loosely linked mutations, i.e., mutations in a large genomic region, 

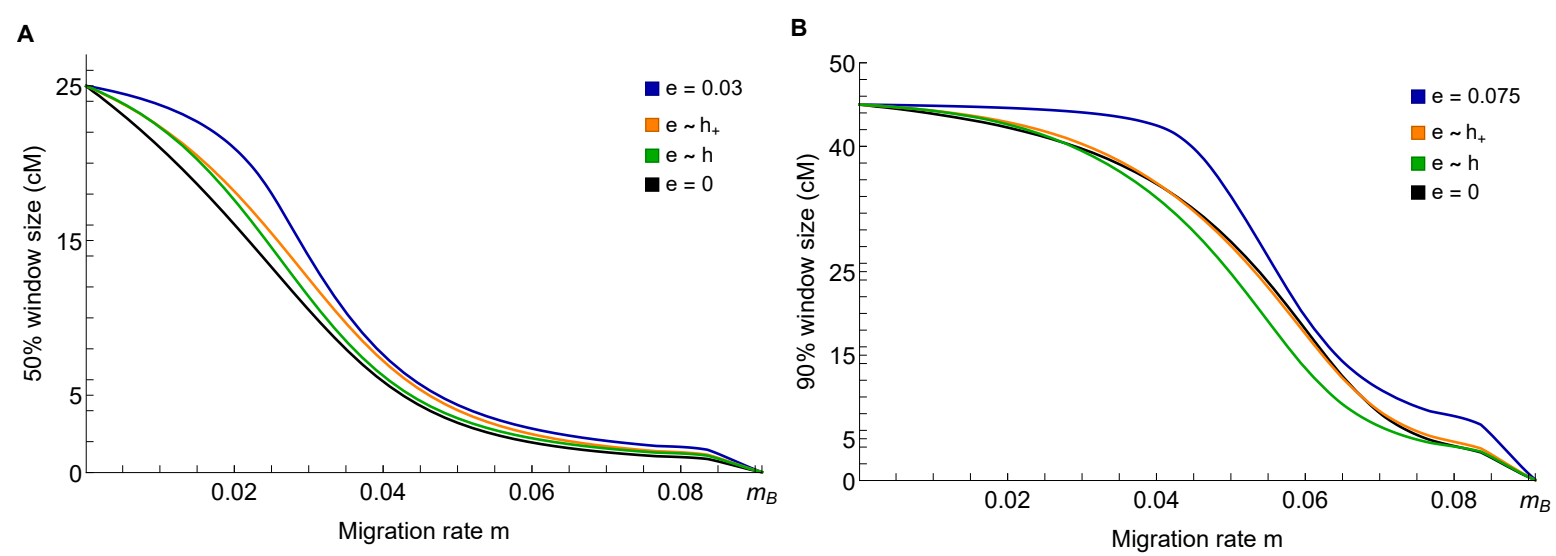

Figure 7: The size of the window within which $50 \%$ (A) or $90 \%$ (B) of all successfully invading linked de novo mutations occur $\left(C_{50}, C_{90}\right)$. In all cases $b=0.1$ and $a$ is drawn from an exponential distribution with mean $\bar{a}=0.01$. The black curves are for additive mutants and the blue curves are for fixed values of epistasis $(e=0.03$ in $\mathrm{A}, e=0.075$ in B). The orange and the green curves show the window sizes if the epistatic effects are drawn from $h_{+}$and $h$, respectively. The units on the ordinate are in centimorgan, thus we identify $r=0.01$ with $1 \mathrm{cM}$. Based on Haldane's mapping function, this is a suitable approximation if $r \lesssim 0.25$.

contribute substantially to the cumulative invasion probability. Because recombination rates are drawn from a uniform distribution on [0,0.5], $C_{50}$ and $C_{90}$ assume the maximum values 0.25 and 0.45 , respectively (at $m=0$ ).

We compute $C_{50}$ and $C_{90}$ numerically and focus, in particular, on the effect of epistasis on the window size. In accordance with intuition, $C_{50}\left(C_{90}\right)$ decreases with increasing $m$ in all observed instances (Figs. 7 and 8). In the absence of epistasis this was also observed for $C_{95}$ by Yeaman et al. (2016). The decline in their Fig. 3 looks much sharper than in our Fig. 7 , but this is deceptive because they used a logarithmic scale. Whereas $C_{50}$ declines nearly linearly in $m$ until $m \approx 0.05$ and then slowly reaches 0 at $m=m_{B} \approx 0.09$ (Fig. $7 \mathrm{~A}$ ), $C_{90}$ declines very slowly until about $m \approx 0.04$ and then rapidly to very small values (Fig. $7 \mathrm{~B})$. In each of the two panels, the decline is somewhat delayed in the case of a high, fixed epistatic value (blue curves), but then occurs more sharply than in the other three cases (no epistasis or epistatic coefficients drawn from either $h$ or $h_{+}$). The reader may keep in mind that the window size is computed based on successfully invading mutants; therefore, the fact that $\bar{\pi}$ decreases as a function of $m$ (Sect. 4) does not directly yield the observed decay of $C_{50}$ or $C_{90}$.

Somewhat surprisingly, Fig. 7 shows that the window size depends only very weakly on epistasis, unless a fixed, high epistatic value is assumed (as for the blue curves). It 
A

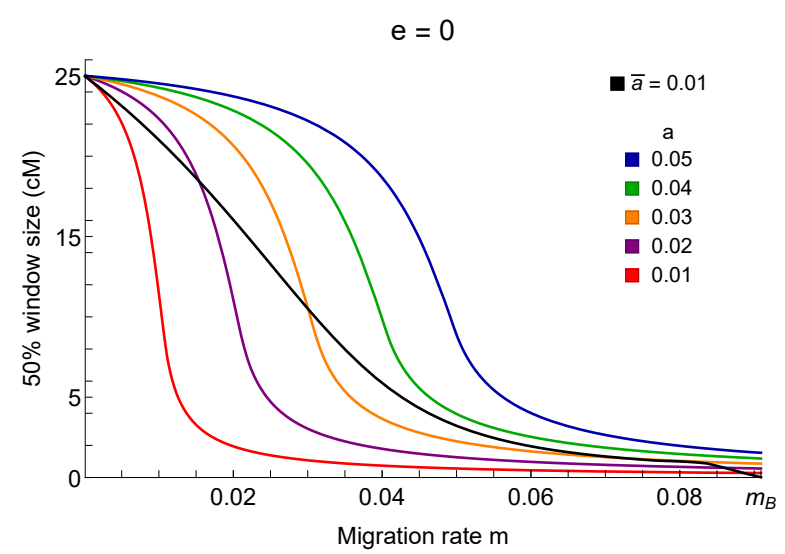

B

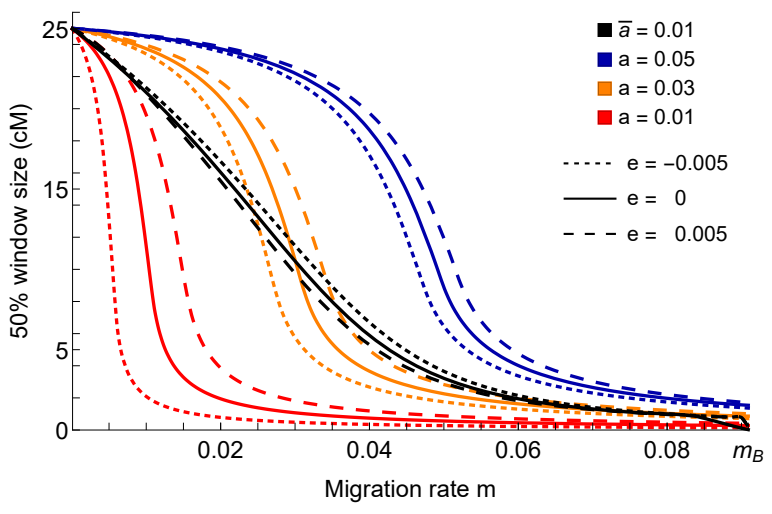

Figure 8: Comparison of $C_{50}$ window sizes for five different values of fixed $a$ and for $a$ drawn from the exponential distribution $f$ with mean $\bar{a}=0.01$. In panel A, absence of epistasis is assumed. In panel $\mathrm{B}$, fixed positive $(e=0.005$, dashed) and fixed negative epistasis ( $e=-0.005$, dotted) epistasis are included and shown for three fixed values of $a$. Note that for $a$ drawn from $f$, the curve with negative epistasis (black, dotted) is higher than that without epistasis (black, solid) as well as that with $e=0.005$ (black, dashed).

may even seem counterintuitive that $C_{50}$ and $C_{90}$ are higher if epistatic effects are drawn from $h_{+}$, which has the negative mean $-\pi \bar{a}$, than if they are drawn from $h$, which has mean 0 .

These observations have a relatively simple explanation. The distribution $h$ has a variance of $2 \bar{a}^{2}$, and the variance of $h_{+}$is about 3 times larger (eqs. 42, 43). Therefore, about $10 \%$ of mutational effects drawn from $h_{+}$are positive (compared to $50 \%$ of those drawn from $h$ ). This is still enough that for both distributions most of the successfully invading mutants have a positive epistatic effect, often close to $\bar{a}$ (see Fig. 6B). The fact that the invasion probability of mutants with epistatic effect drawn from $h_{+}$typically is $1 / 5-1 / 4$ of that of mutants with epistatic effect drawn from $h$ (see Fig. S4) does not directly affect the window size, which is conditioned on successful invasion. Because successful mutants with $e$ drawn from $h_{+}$have a smaller average value than mutants with $e$ drawn from $h$, i.e., $\bar{e}_{+, s}<\bar{e}_{s}$, they have a slightly higher additive effect, i.e., $\bar{a}_{+, s}>\bar{a}_{s}$ (Fig. 6A). Their total average effects $\left(\bar{a}_{s}+\bar{e}_{s}\right)$ are nearly identical except in the high-migration case $m=0.07$ (Fig. 6C), where invasion of loosely linked mutations is already extremely unlikely. In summary, mutants with $e$ drawn from $h_{+}$can invade at a (slightly) higher distance from the polymorphic site than mutants with $e$ drawn from $h$.

Because several factors determine the window size in a complex way if mutational 
effects are drawn from distributions, we studied the window size for fixed $a$ and $e$. Figure 8 shows $C_{50}$ for several choices of $a$ and $e$ and compares it with $C_{50}$ obtained by choosing $a$ from the exponential distribution $f$. The main difference is the much more gradual decline of the black curves ( $a$ drawn from $f$ ) in comparison to the others (fixed $a$ ), which show a sharp decline in a relative small range of recombination rates. In the absence of epistasis (panel A), this sharp decline for fixed $a$ is due to the fact that for increasing migration rate $m$ the probability of invasion is rapidly decreasing as $r$ increases. The much more gradual decline of $C_{50}$, when the values of $a$ are drawn from $f$, is explained by fact that with increasing $r$ successfully invading mutants have increasingly larger additive effects $a$, as is reflected by the increase of $\bar{a}$ shown in Fig. 7A. A similar line of arguments also explains why, in the case of epistasis (Fig. 8B) the order of the black dashed curves (where $a$ is drawn from $f$ ) is reversed relative to the curves with a fixed value of $a$. If $e<0$ then the values of $a$ that lead to invasion are larger than if $e>0$ (Fig. S5C), and larger values of $a$ enable invasion in a bigger neighborhood of the already polymorphic locus.

\section{Discussion}

We have performed an analysis of the effects of epistasis on the fate of a new, weakly beneficial mutation in a haploid population that is exposed to maladaptive gene flow. The mutant, $A_{1}$, arises at a locus $A$ that is linked (recombination rate $r$ ) to an already established migration-selection polymorphism $E_{B}$ at a locus $B$. The existence of the polymorphism requires that the immigration rate $m$ of the deleterious allele $B_{2}$ is bounded by the constant $m_{B}=b /(1+b)$, where $b$ is the selective advantage of $B_{1}$. In particular, we characterized the region of the parameter space, in which the de novo mutation $A_{1}$ can survive the stochastic phase after its occurrence (Propositions 1, 2, 4, 5). The first two propositions apply to evolutionary forces of arbitrary strength, whereas the two others are derived under the assumption of weak evolutionary forces. This yields simple and intuitive conditions for invasion. In each case, invasion is impossible if $a+e<0$, where $a>0$ is the mutant's effect on background $B_{2}$ and $a+e$ that on $B_{1}$. In this parameter regime, $A_{1}$ exhibits sign epistasis, i.e., the mutation $A_{1}$ is deleterious in the presence of $B_{1}$, but beneficial on the $B_{2}$ background (Weinreich et al., 2005). Therefore, sign epistasis prevents establishment of $A_{1}$ independently of the (total) strength of the evolutionary forces. This is in line with the known effect of sign epistasis to constrain the selective availability of mutational trajectories to genotypes of high fitness, as laid out by Weinreich et al. (2005). 
If the strength $e$ of epistasis exceeds $-a$ but is below a certain bound $e_{r}$ or $e_{m}$, which depends on $b$ and on $r$ or $m$, respectively, then invasion is possible below a critical value of $m$ or $r$, respectively. If $e>e_{r}$ or $e>e_{m}$, then invasion of $A_{1}$ in an equilibrium population at $E_{B}$ is possible for every admissible $m$ or $r$, respectively (Propositions 1 or $2)$. The critical values $e_{r}$ and $e_{m}$ can be positive or negative. If they are positive, then an additive mutant $A_{1}(e=0)$ can invade only for sufficiently small $m$ or $r$, respectively (Fig. 1). In summary, for fixed $b$, increasing $e$ (and of course increasing $a$ ) widens the range of parameters in which invasion can occur.

It is also important to determine if $A_{1}$ can enter the population exclusively through the single-locus polymorphism $E_{B}$, or if there are further possibilities. If allele $B_{1}$ is absent from the island population, then, of course, $A_{1}$ can invade the resident population consisting of $A_{2} B_{2}$ individuals if $m<\frac{a}{1+a}=m_{A}$. If $B_{1}$ is present and $0<m<m_{B}$, then the single locus polymorphism exists and invasion of allele $A_{1}$ is possible under the conditions derived in Propositions 1 and 2. If $m>m_{B}>m_{A}$ (which holds because we assume $a<b$ ), then the island population is swamped by the continental type $A_{2} B_{2}$, which eventually becomes fixed.

A full treatment of the deterministic haploid two-locus two-allele dynamics under the weak forces assumption with continent-to-island migration can be found in Bank et al. (2012). They showed that the maximum possible number of internal equilibria is three and at most one of them is stable. This stable equilibrium corresponds to a so-called Dobzhansky-Muller incompatibility (DMI), which is the key to explain the evolution of intrinsic postzygotic isolation. The convergence to this DMI is the most likely outcome of the long-term dynamics after successful invasion, provided the population size is sufficiently high.

Branching process theory yields a system of equations from which the probability of invasion can be computed. Because they are transcendental, only numerical but no explicit analytical solution is possible. However, by assuming that the branching process is slightly supercritical, analytical approximations become available (Athreya, 1993; Haccou et al., 2005, and Sect. 4). Although the explicit approximations are complex and not directly informative (File S3.2), they are useful to investigate the dependence of the invasion probability on the model parameters (Sect. 4.1). We find that the invasion probability is always increasing with decreasing $m$ and with increasing $a$ or $e$. For a wide region of parameters, it also decreases with increasing $r$. Nevertheless, there is a significant parameter region in which a non-zero optimal recombination rate occurs, i.e., where the invasion probability is maximized at an $r>0$ (Sect. 4.2). This 
phenomenon was already observed in the diploid case without epistasis by Aeschbacher and Bürger (2014). However, with positive epistasis a non-zero optimal recombination rate occurs for a wider range of parameters and the optimum may be even at $r=\frac{1}{2}$ (Figs. 2B and S1).

Related results have been found in two-locus models of panmictic populations by Ewens (1967), who used a branching process approximation to study invasion, and by Lessard and Kermany (2012), who used an ancestral selection-recombination graph to study fixation of new mutants. The latter authors showed that in a large (but finite) population, the ultimate fixation probability of a mutant increases if it is in positive epistasis with another beneficial, already segregating mutant, or if it is itself beneficial and there is no epistasis. These results were shown for weak recombination and weak selection and confirm the Hill-Robertson effect. In our branching process model, the Hill-Robertson effect doesn't seem to be responsible for the non-zero optimal recombination rate, because the population of resident alleles is infinite by assumption. In our model, low recombination favors the spreading of the mutant if it initially occurs on the good background $\left(B_{1}\right)$, whereas strong recombination is necessary for successful invasion if it occurs on the deleterious background $\left(B_{2}\right)$. However, if the mutant occurs on the bad background, strong recombination helps only if the effect $a+e$ on the good background is sufficiently large that invasion can occur without the help of very tight linkage, i.e., if $a+e>b-a$; see (40).

Local adaptation to a new environment is often modeled by assuming that a quantitative trait is subject to Gaussian stabilizing selection, where the population mean is displaced from the fitness optimum. If this displacement is large, the fitness landscape around the mean will be convex in this setting. In particular, every mutation that brings the population closer to the optimum is beneficial and positive epistasis will accelerate adaptation. For this situation our parametrization in equation (1) is well suited. If the population is not far from the optimum, the fitness landscape will be concave and the maximum fitness cannot be exceeded by new mutations. In this situation, the parameterization in (36) may be most appropriate. If mutations of large effect can overshoot the optimum, then, depending on the background on which they occur, they may reduce fitness. In such a case, negative epistasis may be beneficial for invasion. This effect is demonstrated in Fig. 4, where the highest invasion probability occurs for negative epistasis, especially if linkage is weak (green curves). Once $e<-a$, invasion is no longer possible in our model (Proposition 1). Obviously, our model was not designed to study invasion close to a fitness optimum. 
Because, in general, neither the genetic effects ( $a$ and $e$ ) of mutants nor their recombinational distance $(r)$ from the existing polymorphism are known, especially not a priori, in Sect. 5 we studied expected invasion probabilities if $a$, e, and $r$ are drawn from probability distributions. We assume that the distribution $f$ of additive fitness effects is exponential with mean $\bar{a}$. Recombination rates are drawn from a uniform distribution.

The distribution of epistasis (in fitness) depends crucially on the relative position of the population with respect to the optimum on the fitness landscape. We use two different ones. The normal distribution $h$, which has mean 0 (see eq. 42 ), was derived from Fishers geometric model for the epistatic interaction between arbitrary mutations (Martin et al., 2007). This may be only of limited suitability for our purposes, because we model epistasis as the interaction between $A_{1}$ and $B_{1}$, which both are advantageous. To account for the interaction of beneficial mutations, we use $h_{+}$, a normal distribution with a negative mean (see eq. 43). This distribution was also derived from Fisher's geometric model by considering the interactions of beneficial mutations (Blanquart et al., 2014). In the derivations of $h$ and of $h_{+}$, it was assumed that the population is far away from the optimum.

We found that a change in the recombination rate affects the invasion probability in a similar way across different epistatic values. Drawing the recombination rate from a uniform distribution again has a similar effect across different epistatic values (Fig. 5). Thus, recombination and epistasis affect the invasion probability essentially independently.

Averaging over epistasis has more subtle consequences for the invasion probability which depend on the properties of the distribution of epistasis. The effects of averaging over $h$ or $h_{+}$can be summarized as follows. For each degree of linkage, the larger share of positive epistatic values in the distribution of $h$ leads to a general increase of the invasion probability compared to the non-epistatic case. The opposite is true if $e$ is distributed according to $h_{+}$with a negative mean. These facts are apparent in Fig. $5 \mathrm{D}$, which shows the ratios of the invasion probability with and without epistasis for the same degree of linkage. Comparison of curves with different degree of linkage in Fig. 5 shows that an effect observed already in Fig. 3 (for fixed values of $e$ and $r$ ) extends to distributions of the parameters $r$ and $e$. For small values of $m$, a larger fraction of positive epistatic values is more efficient in boosting the invasion probability compared to a non-epistatic scenario with tighter linkage. This is reversed above an intermediate value of $m$ when non-epistatic linked mutations contribute more to the 
invasion probability (e.g., compare the black solid curve with either the orange dotted or the dashed green curves in Fig. 5B).

Finally, we investigated the effect of epistasis on the size of the genomic window, or neighborhood, around an already existing polymorphism in which $50 \%$ or $90 \%$ of all successfully invaded linked de-novo mutations occur. For fixed additive and epistatic effects, a sharp decline of the window size occurs in small range if migration rates, whereas the decline is much more gradual if effects are drawn from a distribution (Figs. 7, 8). If the additive fitness value is drawn from an exponential distribution, then drawing the epistatic value from either of our two distributions does not substantially affect $C_{50}$ and $C_{90}$ in comparison to $e=0$. This is in contrast to the distinctively different effects of these distributions on the average invasion probability (Fig. 5B). The reason is that $C_{50}$ and $C_{90}$ are computed by conditioning on successful invasion. Indeed, the average additive and average total effects of successfully invading mutations are very similar in both cases except for the largest migration rate (Fig. 6), but the respective invasion probabilities may differ by about one order of magnitude (Fig. S4). In summary, unless epistasis is consistently positive and very strong, on average it seems to have a weak effect on the size of genomic islands.

Because the sign and the strength of epistasis affect the invasion probability of mutants substantially, they will also strongly affect the time horizon in which adaptation occurs and clusters of beneficial mutations, or genomic islands, build up. Analogously, invasion probabilities may decline by several orders as the migration rate increases (Fig. S4), and therefore the emergence of very tightly linked clusters, as predicted by the small window sizes at higher migration rates, will occur only on very long time scales.

The average invasion probability and the magnitude of effects of successful mutants are also strongly affected by linkage. Mutants tightly linked to the polymorphic site have a relatively high invasion probability, which depends relatively weakly on the migration rate. In addition, their (average) effects are much smaller than for loosely linked mutations, which may reduce the possibility of detection. For moderate to strong migration, the invasion probability declines by several orders of magnitude as $r$ increases from small values (say, $\leq 0.01$ ) to large values (close to 0.5 ). The waiting time for the establishment of such a mutation will be inversely proportional to its invasion probability. Furthermore, with increasing $r$, the mean effect of successfully invading mutations increases substantially (up to about four fold; see Fig. 6A). Thus, beneficial mutants will become established in the vicinity of locus B on a relatively short time 
scale, but will have predominantly small effects. By contrast, establishment of loosely linked or unlinked beneficial mutants will occur on a much longer time scale, but their effects will tend to be relatively large. These complex dependencies may severely affect the time scale on which clusters of locally beneficial mutations build up, as well as their detection.

Acknowledgments. We thank Simon Aeschbacher for very helpful discussions and Sabin Lessard for helpful comments on an earlier version.

Financial support by the Austrian Science Fund (FWF) through the Vienna Graduate School of PopulationGenetics (GrantDK W1225-B20) to MP is gratefully acknowledged.

\section{References}

Aeschbacher S. and Bürger R. 2014. The effect of linkage on establishment and survival of locally beneficial mutations. Genetics, 197, 317-336.

Athreya K.B. 1993. Rates of decay for the survival probability of a mutant gene II The multitype case. J. Math. Biol. 32, 45-53.

Bank C., Bürger R., Hermisson J. 2012. Limits to parapatric speciation: DobzhanskyMuller incompatibilities in a continent-island model. Genetics, 191, 845-863.

Bataillon T., Bailey S.F. 2014. Effects of new mutations on fitness: insights from models and data. Ann. N.Y. Acad. Sci. 1320, 76-92.

Blanquart F., Achaz G., Bataillon T. and Tenaillon O. 2014. Properties of selected mutations and genotypic landscapes under Fishers geometric model. Evolution 68$12,3537-3554$.

Bürger R. 2014. A survey of migration-selection models in population genetics. Discrete Continuous Dynamics Systems B, 883-959.

Ewens W. J. 1967. The probability of fixation of a mutant: the two-locus case. Evolution $21,532-540$.

Ehrenreich I. M. 2017. Epistasis: Searching for interacting genetic variants using crosses. G3 7, 1619-1622.

Feder J. L., Nosil P. 2010. The efficacy of divergence hitchhiking in generating genomic islands during ecological speciation. Evolution 64, 1729-1748. 
Felsenstein J. 2019. Theoretical Evolutionary Genetics. Version accessed in April 2020 of http://evolution.genetics.washington.edu/pgbook/pgbook.html

Fisher R.A. 1930. The Genetical Theory of Natural Selection. Oxford: Claredon Press.

Gao H., Granka J. M. and Feldman M.W. 2010. On the classification of epistatic interactions. Genetics 184, 827-837.

Haccou P., Peter J., and Vatutin V.A., 2005. Branching Processes: Variation, Growth, and Extinction of Populations, Vol. 5, Cambridge Studies in Adaptive Dynamics. Cambridge University Press, New York.

Haldane, J.B.S., 1927. A mathematical theory of natural and artificial selection. V. Selection and mutation. Math. Proc. Camb. Philos. Soc. 23: 838-844.

Haldane, J.B.S., 1930. A mathematical theory of natural and artificial selection. VI. Isolation. Math. Proc. Camb. Philos. Soc. 26: 220-230.

Harr B. 2006. Genomic islands of differentiation between house mouse subspecies. Genome Research 16, 730-737.

Harris T.E. 1963. The Theory of Branching Processes, Vol. 119, Die Grundlehren der Mathematischen Wissenschaften, Ed. 1. Springer-Verlag, Berlin.

Lessard S. and Kermany A. R. 2012. Fixation probability in a two-locus model by ancestral recombination-selection graph. Genetics 190, 691-707.

Martin G., Elena S. F. and Lenormand T. 2007. Distribution of epistasis in microbes fit predictions from a fitness landscape model. Nature Genetics 39, 555-560.

Nosil P., Funk D.J., Ortiz-Barrientos D. 2009. Divergent selection and heterogeneous genomic divergence. Molecular Ecology 18, 375-402.

Orr H.A. 2010. The population genetics of beneficial mutations. Phil. Trans. R. Soc. B $365,1195-1201$.

Rice D.P., Good B.H. and Desai M.M. 2015. The evolutionarily stable distribution of fitness effects. Genetics 200, 321-329.

Schoustra S., Hwang S., Krug J. and de Visser J.A.G.M. 2016. Diminishing-return epistasis among random beneficial mutations in a multicellular fungus.Proc. R. Soc. B 283: 20161376 
Turner T.L., Hahn M.W., Nuzhdin S.V. 2005. Genomic islands of speciation in anopheles gambiae. PLoS Biology, 3, 1572-1578.

Weinreich D.M., Watson R.A. and Chao L. 2005. Perspective: sign epistasis and genetic constraint on evolutionary trajectories. Evolution 59, 1165-1174.

Wolfram Research, Inc. 2020. Mathematica, Version 12.1, Champaign, IL

Wright S. 1931. Evolution in Mendelian populations. Genetics 16, 97-159.

Yeaman S., Aeschbacher S., Bürger R. 2016. The evolution of genomic islands by increased establishment probability of linked alleles. Mol. Ecology 25, 2542-2558.

Yeaman S., Whitlock M.C. 2011. The genetic architecture of adaptation under migration-selection balance. Evolution 65, 1897-1911. 


\section{Appendix}

\section{A Approximations of the invasion probability $\pi_{i}(\epsilon)$ in $(25)$}

Here, we derive the expression $\beta(\epsilon)$ given in (26), which plays a key role in the approximation of the invasion probability $\pi_{i}(\epsilon)$ in $(25)$, derived for a supercritical branching process by Athreya (1993) (see also Haccou et al., 2005, pp. 126-128). In addition, we show that the expression $\beta_{\bullet}(\epsilon)$ given by Aeschbacher and Bürger (2014) (eq. (64) in their Supporting Information, File S1) satisfies $(27)$ and $\beta_{\bullet}(\epsilon) \leq \beta(\epsilon)$. However, if $r>0$, in general the difference between $\beta_{\bullet}(\epsilon)$ and $\beta(\epsilon)$ is of order $O(\epsilon)$, so that $\beta_{\bullet}(\epsilon)$ does not always yield a first-order approximation in $\epsilon$ of $\pi_{i}(\epsilon)$. Nevertheless, for small values of $r, \beta_{\bullet}(\epsilon)$ often yields a more accurate approximation of $\pi_{i}(\epsilon)$ than $\beta(\epsilon)$. In particular, in contrast to $\beta(\epsilon)$, it has the feature that the invasion probability may be maximized at a positive $r$.

We assume independent Poisson offspring distributions. Our starting point is the expression (5.81) in (Haccou et al., 2005, pp. 127), which in our notation from Section 4 reads

$$
\beta(\epsilon)=\sum_{k=1}^{2} u_{k} \operatorname{Var}\left[\sum_{j=1}^{2} v_{j} \xi_{k j}\right]+\lambda(\lambda-1) \sum_{j=1}^{2} u_{j} v_{j}^{2},
$$

where $\xi_{k j}$ are the Poisson variates for the offspring distribution, so that the expected number of offspring of type $j$ of a type $k$ parent is $\mathrm{E}\left[\xi_{k j}\right]=\lambda_{k l}$. Therefore, $\operatorname{Var}\left[\xi_{k j}\right]=\lambda_{k l}$. For notational simplicity, we omit the dependence of $\lambda, u$, and $v$ on $\epsilon$. Recall from Section 4 that the entries of the mean matrix $\mathbf{M}$ are the $\lambda_{k l}$, and that $\lambda$ is its principal eigenvalues.

We observe that

$$
\begin{aligned}
\sum_{k=1}^{2} u_{k} \operatorname{Var}\left[\sum_{j=1}^{2} v_{j} \xi_{k j}\right] & =\sum_{k=1}^{2} u_{k} \sum_{j=1}^{2} v_{j}^{2} \operatorname{Var}\left[\xi_{k j}\right] \\
& =\sum_{j=1}^{2} v_{j}^{2} \sum_{k=1}^{2} u_{k} \lambda_{k j} \\
& =\lambda \sum_{j=1}^{2} v_{j}^{2} u_{j}
\end{aligned}
$$

because $u$ is the left principal eigenvector of M. Substituting (A.2) into (A.1) yields $(26)$. 
Aeschbacher and Bürger (2014, eq. (64) in File S1 of their Supporting Information) had obtained an erroneous expression (because of a missing superscript), which after using $u \mathbf{M}=\lambda u$ can be written as in (27). Now we show that $\beta \bullet(\epsilon) \leq \beta(\epsilon)$. It suffices to show $\sum_{j=1}^{2} u_{j} v_{j}^{2} \geq 1$. From the normalization (24), we obtain $u_{2}=1-u_{1}$ and $v_{2}=\left(1-u_{1} v_{1}\right) /\left(1-u_{1}\right)$. Therefore,

$$
u_{1} v_{1}^{2}+u_{2} v_{2}^{2}=u_{1} v_{1}^{2}+\left(1-u_{1}\right) \frac{\left(1-u_{1} v_{1}\right)^{2}}{\left(1-u_{1}\right)^{2}}=1+\frac{u_{1}\left(1-v_{1}\right)^{2}}{1-u_{1}} \geq 1 .
$$

It is easily shown that $\sum_{j=1}^{2} u_{j} v_{j}^{2}-1=O(\epsilon)$, but not necessarily of order $O\left(\epsilon^{2}\right)$. Therefore, $\beta(\epsilon)$ and $\beta_{\bullet}(\epsilon)$ may differ to first order in $\epsilon$. Therefore, (25) shows that $\bar{\pi}_{\bullet}(\epsilon)$ does not necessarily provide an approximation of $\pi_{i}(\epsilon)$ to order $\epsilon$.

However, if $r=0, r=r^{*}, m=0$, or $m=m^{*}$, we have $\beta \bullet(\epsilon)=\beta(\epsilon)$. Indeed, if $r=0$, then $\mathbf{M}$ is a diagonal matrix with principal eigenvalue $\lambda=1+(a+e) /(1+b)$ and corresponding left and right eigenvectors $u=(1,0)$ and $v=(1,0)^{T}$. If $m=0$, then $\mathbf{M}$ is a triagonal matrix with principal eigenvalue $\lambda=1+(a+e) /(1+b)$ and corresponding eigenvectors $u=(1,0)$ and $v=(1,(1+a) r /(b+e+(1+a) r))^{T}$. In both cases $(27)$ shows that

$$
\beta_{\bullet}(\epsilon)=\beta(\epsilon)=\frac{(1+a+b+e)^{2}}{(1+b)^{2}}=\lambda^{2} .
$$

Finally, if $r=r^{*}$ or $m=m^{*}$, then $\epsilon=0, \lambda=1$ and $\pi_{i}(0)$ is independent of $\beta(0)$ by (25).

Figures 2 and S2 compare the approximations $\bar{\pi}_{\bullet}(\epsilon)$ and $\bar{\pi}(\epsilon)$ with the true mean fixation probability $\bar{\pi}$ and highlight their accuracy.

\section{B Explicit approximations for small $m$ or small $r$}

Because $\lambda(\epsilon)=1+\rho(\epsilon)=1$ if $r=r^{*}$ or $m=m^{*}$, we have $\pi_{1}(\epsilon)=\pi_{2}(\epsilon)=0$ in these cases. In addition, for small $r$ and based on $\beta(\epsilon)$, we obtain the approximation

$$
\begin{aligned}
\left(\pi_{1}(\epsilon), \pi_{2}(\epsilon)\right)= & \frac{2(1+b)(a+e)}{(1+b+a+e)^{2}}\left(1-\frac{(1+b-a-e) m r}{b(a+e)(1-m)}, \frac{(1+a)[b(1-m)-m] r}{b(b+e)(1-m)}\right) \\
& +O\left(r^{2}\right)
\end{aligned}
$$

(File S3, Sect. 6). From (6), (7) and (B.1) we obtain for the mean invasion probability close to $r=0$ :

$$
\begin{aligned}
\bar{\pi}(\epsilon)= & \frac{2(1+b)[b(1-m)-m]}{b^{2}(b+e)(1+b+a+e)^{2}} \cdot(b(b+e)(a+e) \\
& \left.\quad-\frac{\left[b-a+b\left(b-2 a-e-a^{2}-a e\right)-(a+e)^{2}\right] m r}{1-m}\right)+O\left(r^{2}\right)
\end{aligned}
$$


The leading-order term can be rewritten as in (29), and the term $[b-a+b(b-2 a-$ $\left.\left.e-a^{2}-a e\right)-(a+e)^{2}\right]$ determines $a^{*}$ in $(37)$, hence whether the derivative of $\bar{\pi}(\epsilon)$ with respect to $r$ is positive or negative.

The corresponding approximation for $\bar{\pi}_{\bullet}(\epsilon)$ is (File S3, Sect. 6)

$$
\begin{aligned}
\bar{\pi}_{\bullet}(\epsilon)= & \frac{2(1+b)[b(1-m)-m]}{b^{2}(b+e)(1+b+a+e)^{2}} \cdot(b(b+e)(a+e) \\
& \left.\quad-\frac{\left[b-2 a-e+b\left(b-3 a-2 e-a^{2}-a e\right)-(a+e)^{2}\right] m r}{1-m}\right)+O\left(r^{2}\right)
\end{aligned}
$$

and $a_{\bullet}^{*}$ in (38) is obtained by solving $b-2 a-e+b\left(b-3 a-2 e-a^{2}-a e\right)-(a+e)^{2}=0$.

For small $m$, the first-order approximation of $\bar{\pi}(\epsilon)$ is more complicated, so we give only the leading term:

$$
\left(\pi_{1}(\epsilon), \pi_{2}(\epsilon)\right)=\frac{2(1+b)(a+e)}{(1+b+a+e)^{2}}\left(1, \frac{(1+a) r}{b+e+r(1+a)}\right)+O(m) .
$$

For $\bar{\pi}(\epsilon)$, we obtained the following first-order approximation in $m$ near $m=0$ :

$$
\begin{aligned}
\bar{\pi}(\epsilon)= & \frac{2(1+b)(a+e)}{(1+b+a+e)^{2}} \\
& -m \frac{2(1+b)(b+e)}{b(1+b+a+e)^{2}(b+e+r+a r)^{3}}\left[A_{0}+A_{1} r+A_{2} r^{2}+A_{3} r^{3}\right],
\end{aligned}
$$

where

$$
\begin{aligned}
& A_{0}=(1+b)(a+e)(b+e)^{2}, \\
& A_{1}=(b+e)\left[\left(2 a^{2}+b\right)(1+b)+(3+2 b+a+2 a b-e) e+a(2+b)\right], \\
& A_{2}=(1+a)\left[2 b^{2}+e(2-3 a+a b-3 e)+b\left(2-2 a+a^{2}\right)\right], \\
& A_{3}=(1+a)^{2}(1-a+b-e) .
\end{aligned}
$$

In the supporting material File $\mathrm{S} 3$, Sect. 4 , we show that $\bar{\pi}(\epsilon)$ is decreasing for small $m$. We do this by showing that $A_{0}+A_{1} r+A_{2} r^{2}+A_{3} r^{3}>0$, if $0<r<1 / 2$.

The corresponding approximations and the verification of the negative slope close to $m=0$ for $\bar{\pi}_{\bullet}(\epsilon)$ are given in File S3, Sects. 4,6 
bioRxiv preprint doi: https://doi.org/10.1101/2021.04.30.442097; this version posted April 30, 2021. The copyright holder for this preprint

(which was not certified by peer review) is the author/funder, who has granted bioRxiv a license to display the preprint in perpetuity. It is made available under aCC-BY-NC 4.0 International license.

\section{Explicit approximations for weak evolutionary forces}

For weak evolutionary forces (Section 3.2), the approximations for $\pi$ become simpler (see File S5, Sect. 3):

$$
\begin{aligned}
& \tilde{\pi}_{1}(\epsilon)=\frac{\sqrt{\tilde{R}}(b(2 a-b+e-r)+\sqrt{\tilde{R}})(b(b+e+r)+\sqrt{\tilde{R}})}{b^{2}\left(2 b^{3}+b^{2}(4 e+3 r)+b\left(2 e^{2}+3 e r+r(r-6 m)\right)+r(\sqrt{\tilde{R}}-6 e m)\right.} \\
& \tilde{\pi}_{2}(\epsilon)=\frac{2 m r-b(b+e+r)+\sqrt{\tilde{R}}}{2 m r} \tilde{\pi}_{1}(\epsilon)
\end{aligned}
$$

and

$$
\begin{aligned}
& \tilde{\pi}_{1 \bullet}(\epsilon)=\frac{(b(2 a-b+e-r)+\sqrt{\tilde{R}})(b(b+e+r)+\sqrt{\tilde{R}})}{2 b \sqrt{\tilde{R}}}, \\
& \tilde{\pi}_{2 \bullet}(\epsilon)=\frac{2 m r-b(b+e+r)+\sqrt{\tilde{R}}}{2 m r} \tilde{\pi}_{1 \bullet}(\epsilon)
\end{aligned}
$$

or shorter:

$$
\tilde{\bar{\pi}}(\epsilon)=\frac{\sqrt{\tilde{R}}(b(2 a-b+e-r)+\sqrt{\tilde{R}})(b(b+e+r)-2 m(b+e)+\sqrt{\tilde{R}})}{b^{2}\left(2 b^{3}+b^{2}(4 e+3 r)+b\left(2 e^{2}+3 e r+r(r-6 m)\right)+r(\sqrt{\tilde{R}}-6 e m)\right.}
$$

and

$$
\tilde{\tilde{\pi}}_{\bullet}(\epsilon)=\frac{(b(2 a-b+e-r)+\sqrt{\tilde{R}})(b(b+e+r)-2 m(b+e)+\sqrt{\tilde{R}})}{2 b \sqrt{\tilde{R}}}
$$

with $\tilde{R}=b\left(b(b+e)^{2}+2(b+e)(b-2 m) r+b r^{2}\right)$. 
bioRxiv preprint doi: https://doi.org/10.1101/2021.04.30.442097; this version posted April 30, 2021. The copyright holder for this preprint (which was not certified by peer review) is the author/funder, who has granted bioRxiv a license to display the preprint in perpetuity. It is made available under aCC-BY-NC 4.0 International license.

\section{SI figures}

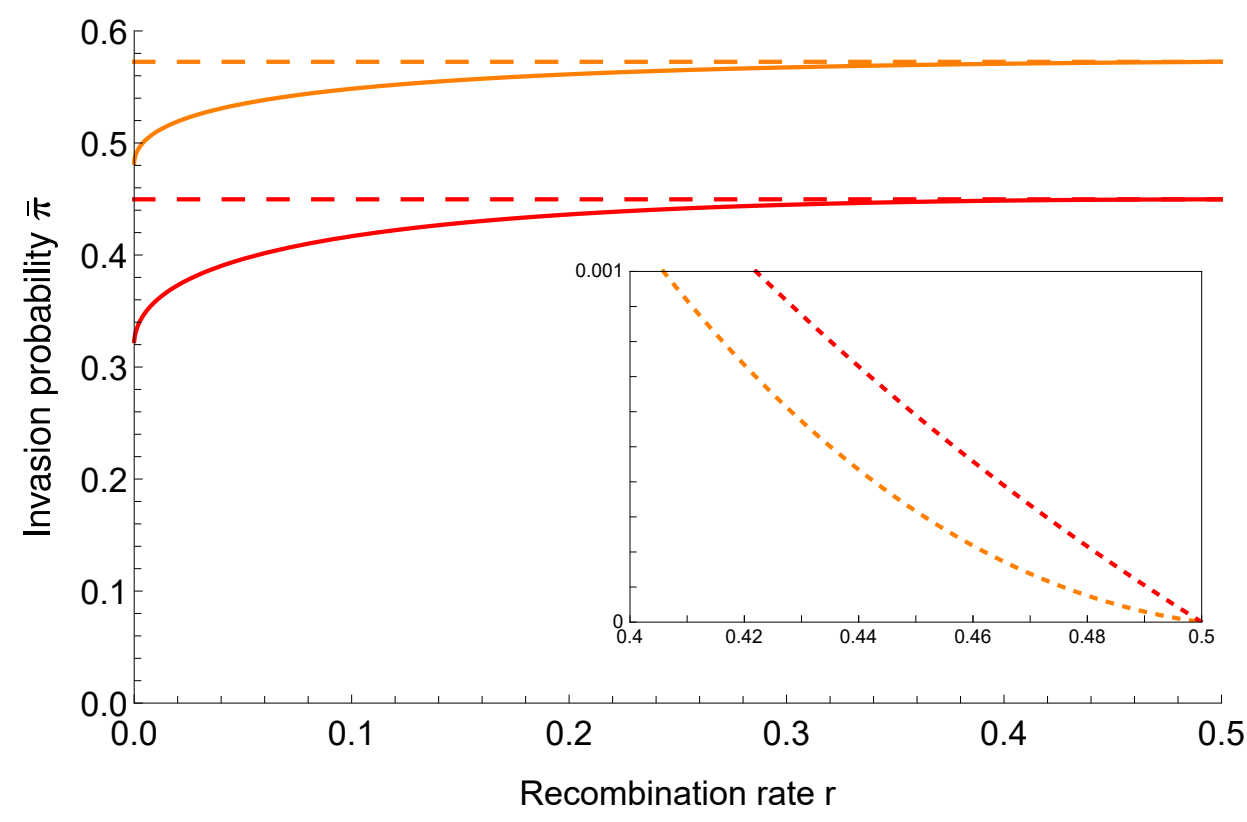

Figure S1: The invasion probability as an increasing function of the recombination rate. The dashed curves are for $r=0.5$, which is in this case the optimum. The orange curves have the lower migration rate $m=0.03$, whereas $m=0.05$ for the red curves. The other values are $a=0.09, b=0.1$ and $e=0.75$. The inset shows the difference between the curves variable in $r$ and those with $r=0.5$ for large $r$. 
bioRxiv preprint doi: https://doi.org/10.1101/2021.04.30.442097; this version posted April 30, 2021. The copyright holder for this preprint (which was not certified by peer review) is the author/funder, who has granted bioRxiv a license to display the preprint in perpetuity. It is made available under aCC-BY-NC 4.0 International license.

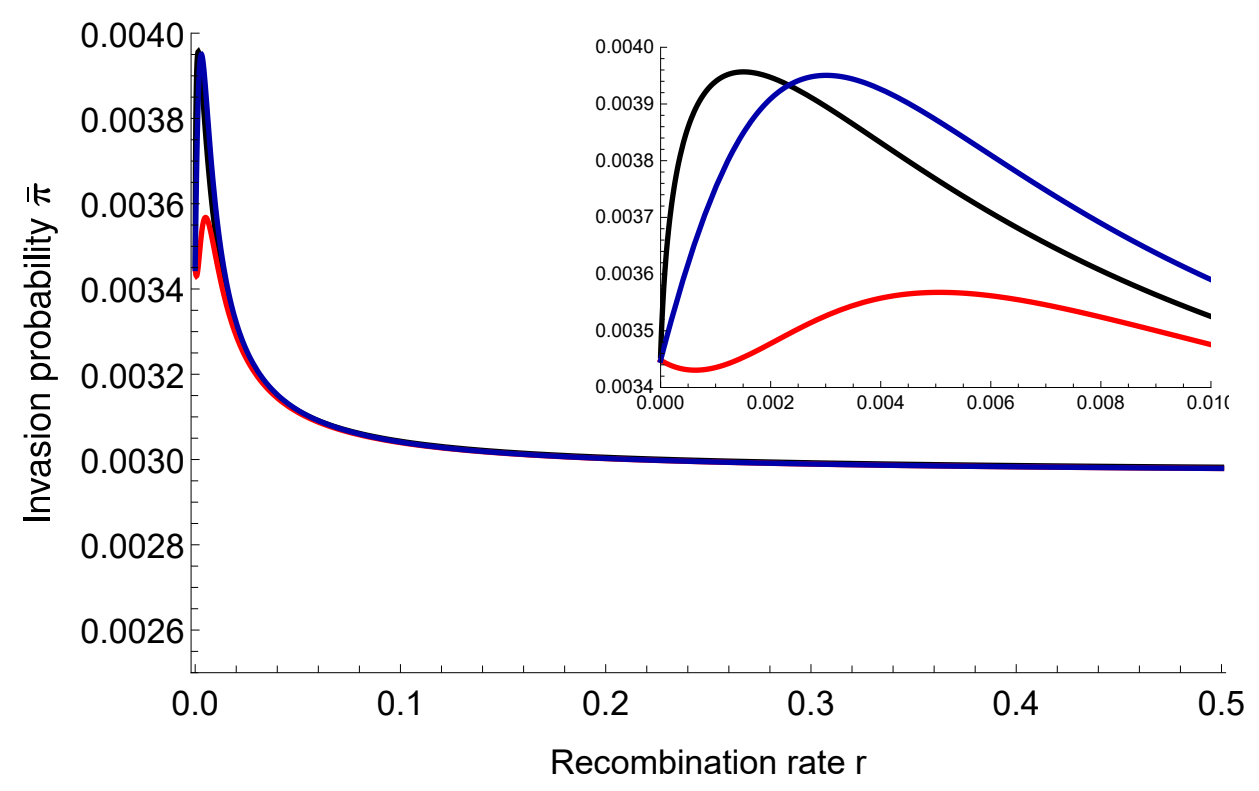

Figure S2: In this Figure, the approximation $\bar{\pi}^{(\epsilon)}$ (red curves) has two local optima, one at $r=0$ and another one for $r>0$. The black curve is the numerical solution, whereas the blue curves correspond to $\bar{\pi}_{\bullet}^{(\epsilon)}$. The parameter values are $a=0.0035, b=$ $0.004, m=0.002$ and $e=0$. Thus, $a^{*}=0.00397$. The inset shows, in detail, what happens for small $r$. 


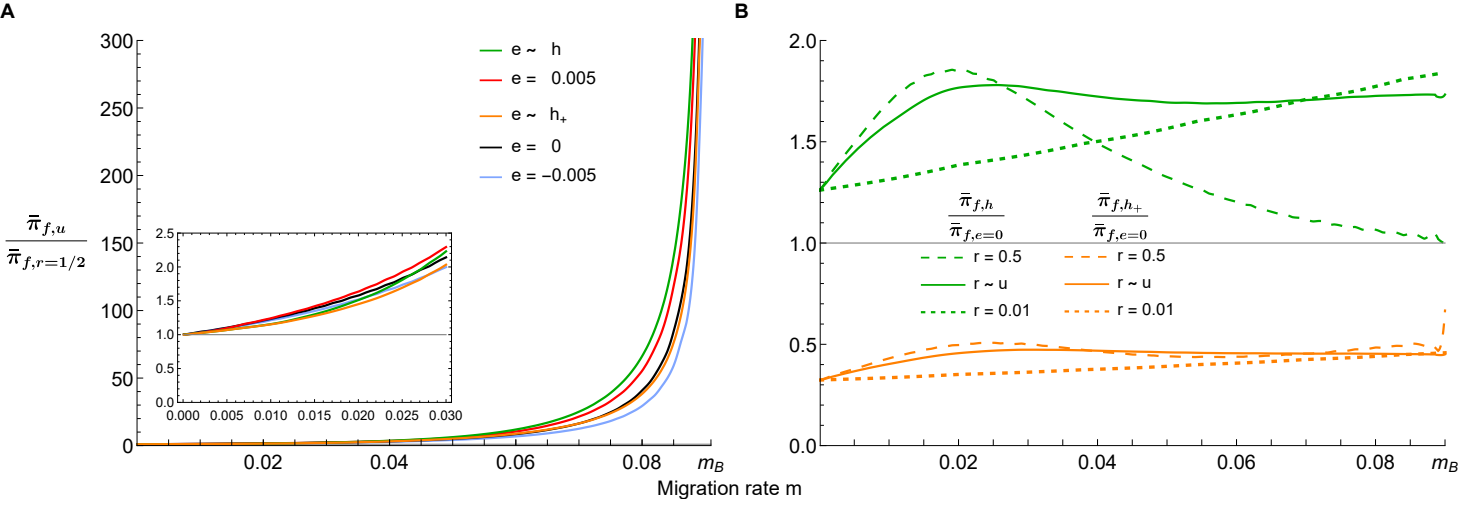

Figure S3: Ratios of averaged invasion probabilities of new mutations as functions of $m$. In all panels the invasion probability is averaged with respect to $f(a)$ with mean $\bar{a}=0.01$. The only constant parameter is $b=0.1$, so that the maximum possible migration rate is $m_{B} \approx 0.091$. For the epistatic values in Fig. $5 \mathrm{~A}$ and the epistasis distributions in Fig. 5B, panel A displays the ratios of the invasion probabilities averaged over $a, r$, and potentially $e$ (i.e., $\bar{\pi}_{f, u, e=-0.005}, \bar{\pi}_{f, u, e=0}, \bar{\pi}_{f, u, e=0.005}, \bar{\pi}_{f, u, h}, \bar{\pi}_{f, u, h_{+}}$), and the corresponding invasion probabilities for unlinked loci (e.g., $\bar{\pi}_{f, h, r=1 / 2}$ ). The line colors in A match those in Figs. 5A,B. Thus, the invasion probabilities shown by the solid curves in panels $\mathrm{A}$ and $\mathrm{B}$ are divided by those of the long-dashed curves of the same color. The curves tend to infinity at the value $m$ at which the dashed curves in Fig. 5A and Fig. 5B reach zero. Panel B shows the ratios of the invasion probabilities averaged over $a$ and $e$ and of those without epistasis. The solid curves are obtained by additional averaging over $r$, i.e., they show $\bar{\pi}_{f, u, h} / \bar{\pi}_{f, u, e=0}$ and $\bar{\pi}_{f, u, h_{+}} / \bar{\pi}_{f, u, e=0}$. Thus, the invasion probability of each of the colored curves in Fig. 5B is divided by the invasion probability of the black curve in Fig. 5B of the same dashing style. The gray horizontal line in A and $\mathrm{B}$ is at 1 to provide a reference for the ratios. 
bioRxiv preprint doi: https://doi.org/10.1101/2021.04.30.442097; this version posted April 30, 2021. The copyright holder for this preprint (which was not certified by peer review) is the author/funder, who has granted bioRxiv a license to display the preprint in perpetuity. It is made available under aCC-BY-NC 4.0 International license.

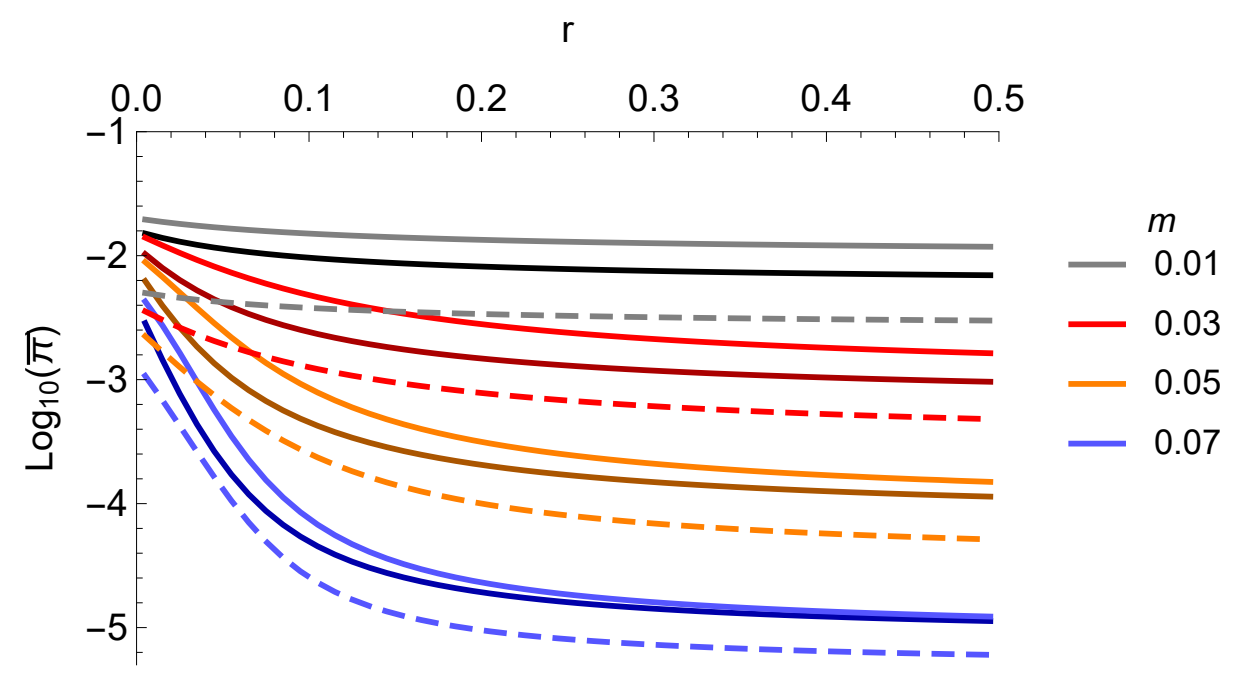

Figure S4: Averaged invasion probabilities corresponding to Fig. 6 as functions of the recombination rate on a logarithmic scale. Shown are $\log _{10} \bar{\pi}_{f, h}$ (bright colors, solid), $\log _{10} \bar{\pi}_{f, h_{+}}$(bright colors, dashed), and $\log _{10} \bar{\pi}_{f, e=0}$ (dark colors, solid). The $\log _{10}$ scale is chosen for improved visibility. The line colors indicate the migration rate (legend). 


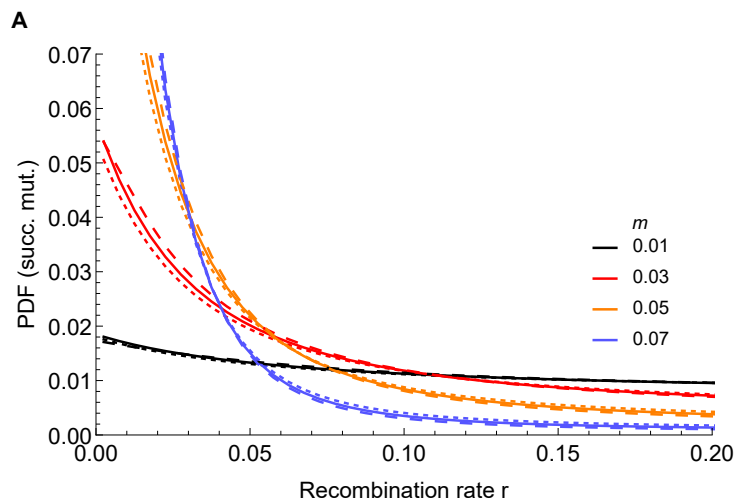

c

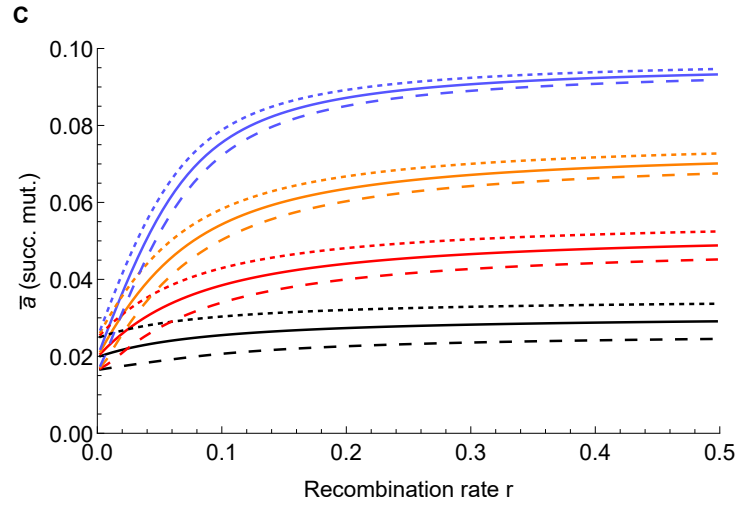

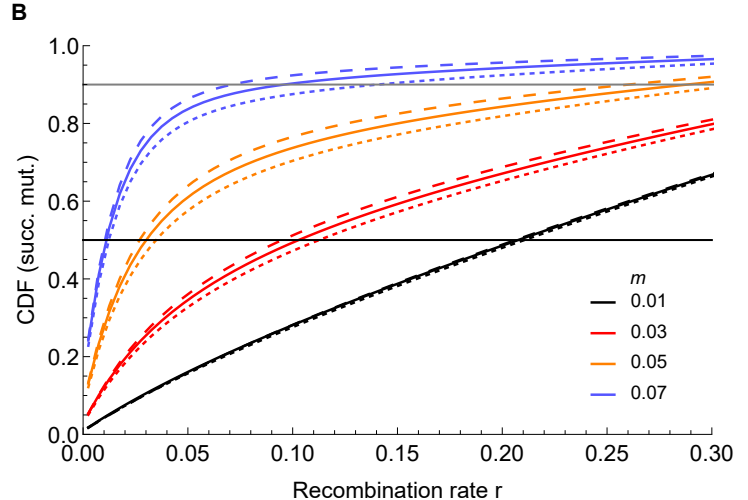

Figure S5: Panel A shows the probability distribution function (PDF) of the case that a mutant can successfully invade the population as a function of $r$, whereas panel $\mathrm{B}$ shows the corresponding CDF. The color code is the same for all three panels and denotes different values of $m$. The panels $\mathrm{A}$ and $\mathrm{B}$ are for the invasion averaged over $a$ with mean $\bar{a}=0.01$, whereas the panel $\mathrm{C}$ shows the average value of $\bar{a}$ with which the invasion is successful. Solid lines represent cases where epistasis is absent. Dashed curves, correspond to positive epistasis, $e=0.005$ and dotted curves correspond to negative epistasis, $e=-0.005$. The horizontal lines in the panel $\mathrm{B}$ are at $50 \%$ and $90 \%$ respectively, from which one can infer $\mathrm{C} 50$ and $\mathrm{C} 90$ respectively. In panel A the blue lines are close to 0.25 at $r=0$ and in panel B the black curves cross the horizontal C90 line at around $r=0.42$. The plots are clipped for better visibility of the main features. 